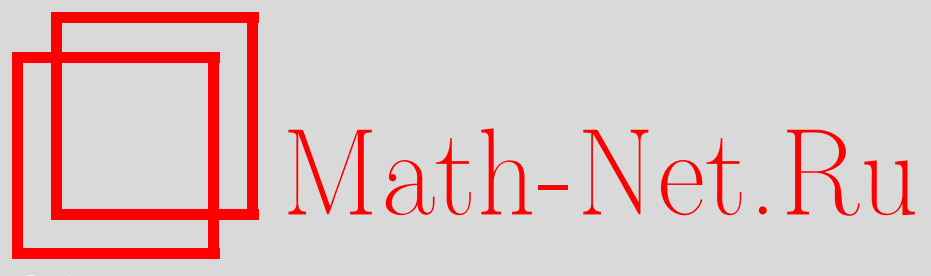

А. С. Холево, Мультипликативность $p$-норм вполне положительных отображений и проблема аддитивности в квантовой теории информации, УМH, 2006, том 61, выпуск 2, 113-152

DOI: https://doi.org/10.4213/rm1709

Использование Общероссийского математического портала Math-Net.Ru подразумевает, что вы прочитали и согласны с пользовательским соглашением http://www . mathnet.ru/rus/agreement

Параметры загрузки:

IP: 34.229 .108 .108

26 апреля 2023 г., 17:29:58

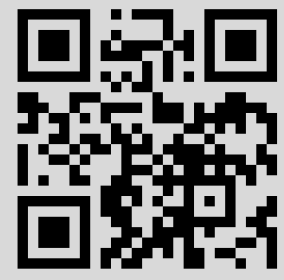




\title{
Мультипликативность $p$-норм вполне положительных отображений и проблема аддитивности в квантовой теории информации
}

\author{
А. С. Холево
}

Проблема аддитивности - одна из наиболее глубоких математических проблем квантовой теории информации. С аналитической точки зрения она тесно связана с вопросом о мультипликативности, относительно тензорных произведений, норм отображений в пространствах операторов, снабженных нормами Шаттена (некоммутативный аналог $l_{p}$-норм). В этой статье дается обзор текущего состояния проблемы.

Библиография: 62 названия.

\section{СОДЕРЖАНИЕ}

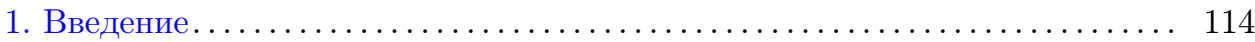

2. Мотивировка из коммутативного анализа.................... 114

3. Некоммутативный случай ................................. 117

3.1. Гипотеза мультипликативности . ................... 117

3.2. Пример: унитальные кубитные каналы .................. 119

3.3. Энтропийные характеристики в.п. отображений и каналов..... 121

3.4. Различные формы гипотезы аддитивности. Формулировка в терминах двойственности выпуклого анализа ............... 124

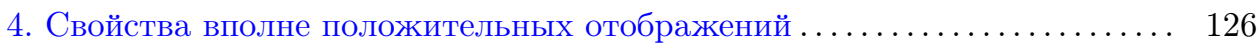

4.1. Обозначения Дирака.................................... 126

4.2. Очищение состояний ............................ 127

4.3. Представления вполне положительных отображений......... 128

5. Мультипликативность/аддитивность для некоторых классов каналов.. 131

5.1. Тождественный канал и связанные с ним каналы ............ 131

5.2. Каналы, разрушающие сцепленность, и комплементарные к ним 132

5.3. Ортогональные выпуклые суммы каналов ................ 138

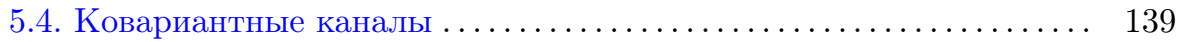

6. Глобальная эквивалентность различных форм аддитивности......... 143

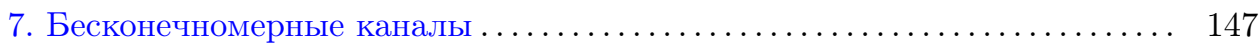

8. Приложение: о $q \rightarrow p$ нормах в.п. отображений ................ 148

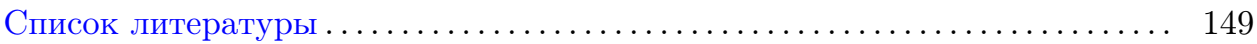

Работа выполнена при поддержке Российского фонда фундаментальных исследований (грант 06-01-0164-а).

(C) А. С. Холево, 2006 


\section{1. Введение}

Проблема аддитивности - одна из наиболее глубоких и до конца еще не решенных математических проблем, возникших с появлением квантовой теории информации (см. [1]-[3] относительно основных фактов и определений этой теории). С аналитической точки зрения, она тесно связана с вопросом о мультипликативности норм отображений в пространствах операторов, снабженных нормами Шаттена (некоммутативный аналог $l_{p}$-норм), относительно тензорных произведений. В этой статье дается обзор текущего состояния этой проблемы.

Мы начинаем в разделе 2 с рассмотрения коммутативного случая, в котором эта проблема имеет простое решение. Затем в разделе 3 дается формулировка проблемы в некоммутативном, конечномерном случае, где также рассматриваются несколько разновидностей гипотезы аддитивности и соотношения между ними. Наиболее важен случай вполне положительных (в.п.) отображений, и мы посвящаем раздел 4 описанию их свойств. В частности, в п. 4.3 излагается конструкция комплементарных отображений, которая приводит к новым примерам мультипликативности/аддитивности. Позитивные результаты для ряда конкретных классов в.п. отображений рассматриваются в разделе 5 , где обсуждается также и важный контрпример. В разделе 6 дается доказательство замечательного результата П. Шора о глобальной эквивалентности всех разновидностей гипотезы аддитивности. В завершающем разделе 7 мы даем краткий обзор работ, относящихся к бесконечномерному случаю.

\section{2. Мотивировка из коммутативного анализа}

Пусть $\mathscr{X}$ - конечное множество, тогда $L(\mathscr{X})$ обозначает комплексное линейное пространство векторов с компонентами, индексированными элементами множества $\mathscr{X}$ (на самом деле $L(\mathscr{X})$ является алгеброй с покомпонентным умножением). Для $p \geqslant 1$ рассмотрим $l^{p}$-нормы вектора $f \in L(\mathscr{X})$

$$
\|f\|_{p}=\left(\sum_{x \in \mathscr{X}}|f(x)|^{p}\right)^{1 / p} .
$$

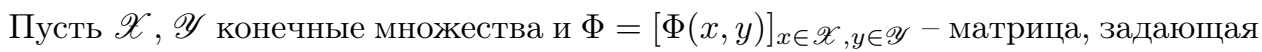
отображение $\Phi: L(\mathscr{X}) \rightarrow L(\mathscr{Y})$ с нормой

$$
\|\Phi\|_{q \rightarrow p}=\max _{f \neq 0} \frac{\|\Phi f\|_{p}}{\|f\|_{q}} .
$$

Рассмотрим два таких отображения $\Phi_{j}: L\left(\mathscr{X}_{j}\right) \rightarrow L\left(\mathscr{Y}_{j}\right) ; j=1,2$, и их тензорное произведение $\Phi_{1} \otimes \Phi_{2}: L\left(\mathscr{X}_{1} \times \mathscr{X}_{2}\right) \rightarrow L\left(\mathscr{Y}_{1} \times \mathscr{Y}_{2}\right)$. Мы здесь используем естественный изоморфизм $L\left(\mathscr{X}_{1} \times \mathscr{X}_{2}\right) \simeq L\left(\mathscr{X}_{1}\right) \otimes L\left(\mathscr{X}_{2}\right), L\left(\mathscr{Y}_{1} \times \mathscr{Y}_{2}\right) \simeq L\left(\mathscr{Y}_{1}\right) \otimes L\left(\mathscr{Y}_{2}\right)$. Известно, что мультипликативность норм отображений

$$
\left\|\Phi_{1} \otimes \Phi_{2}\right\|_{q \rightarrow p}=\left\|\Phi_{1}\right\|_{q \rightarrow p}\left\|\Phi_{2}\right\|_{q \rightarrow p}
$$

имеет место в следующих случаях:

1) $1 \leqslant q \leqslant p$;

2) $\Phi_{1}\left(x_{1}, y_{1}\right) \geqslant 0$ и $1 \leqslant q, p$. 
На самом деле, оба эти результата верны в более общем контексте ограниченных интегральных операторов из $L^{q}(\mathscr{X})$ в $L^{p}(\mathscr{Y})$, где $\mathscr{X}, \mathscr{Y}$ - пространства с мерами; первое утверждение следует тогда из леммы 2 работы [4] и было независимо получено в [5] в случае матриц. Второе утверждение при $\Phi_{1}=\Phi_{2}$ следует из доказательства теоремы 3.2 в [6], хотя доказательство для $\Phi_{1} \neq \Phi_{2}$ аналогично, см. [7]. Неравенство $\geqslant$ в (2) очевидно (достаточно ограничиться функциями вида $f\left(x_{1}, x_{2}\right)=f_{1}\left(x_{1}\right) f_{2}\left(x_{2}\right)$ при минимизации в левой части $(2)$, чтобы получить правую), тогда как доказательство обратного неравенства получается применением неравенства Минковского: для $r \geqslant 1$

$$
\left\{\sum_{x}\left[\sum_{y}|F(x, y)|\right]^{r}\right\}^{1 / r} \leqslant \sum_{y}\left\{\sum_{x}|F(x, y)|^{r}\right\}^{1 / r}
$$

(причем равенство достигается тогда и только тогда, когда $\left.F(x, y)=F_{1}(x) F_{2}(y)\right)$. Покажем, например, как это неравенство используется во втором случае. Имеем

$$
\begin{aligned}
\left\|\left(\Phi_{1} \otimes \Phi_{2}\right) F\right\|_{p}^{p} & =\sum_{y_{1}, y_{2}}\left|\sum_{x_{1}} \sum_{x_{2}} \Phi_{1}\left(x_{1}, y_{1}\right) \Phi_{2}\left(x_{2}, y_{2}\right) F\left(x_{1}, x_{2}\right)\right|^{p} \\
& \leqslant \sum_{y_{1}} \sum_{y_{2}}\left|\sum_{x_{1}} \Phi_{1}\left(x_{1}, y_{1}\right)\right| K\left(x_{1}, y_{2}\right)||^{p}
\end{aligned}
$$

где $K\left(x_{1}, y_{2}\right)=\sum_{x_{2}} \Phi_{2}\left(x_{2}, y_{2}\right) F\left(x_{1}, x_{2}\right)$, поэтому по определению норм (1)

$$
\sum_{y_{2}}\left|K\left(x_{1}, y_{2}\right)\right|^{p} \leqslant\left\|\Phi_{2}\right\|_{q \rightarrow p}^{p}\left[\sum_{x_{2}}\left|F\left(x_{1}, x_{2}\right)\right|^{q}\right]^{p / q}
$$

для всех $x_{1}$. Используя неравенство Минковского с аргументами суммирования $x_{1}, y_{2}$, получаем, что (3) меньше или равно, чем

$$
\begin{aligned}
& \sum_{y_{1}}\left\{\left.\left.\sum_{x_{1}}\left|\sum_{y_{2}} \Phi_{1}\left(x_{1}, y_{1}\right)^{p}\right| K\left(x_{1}, y_{2}\right)\right|^{p}\right|^{1 / p}\right\}^{p} \\
& \leqslant\left\|\Phi_{2}\right\|_{q \rightarrow p}^{p} \sum_{y_{1}}\left\{\sum_{x_{1}} \Phi_{1}\left(x_{1}, y_{1}\right)\left[\sum_{x_{2}}\left|F\left(x_{1}, x_{2}\right)\right|^{q}\right]^{1 / q}\right\}^{p} \\
& \leqslant\left\|\Phi_{1}\right\|_{q \rightarrow p}^{p}\left\|\Phi_{2}\right\|_{q \rightarrow p}^{p}\left[\sum_{x_{1}, x_{2}}\left|F\left(x_{1}, x_{2}\right)\right|^{q}\right]^{p / q} .
\end{aligned}
$$

Нас будет интересовать главным образом специальный случай положительных отображений с нормами $\|\Phi\|_{1 \rightarrow p}$ (для краткости будем называть их просто $p$-нормами), в котором природа мультипликативности наиболее прозрачна. Итак, $\Phi(x, y) \geqslant 0$, откуда, полагая $\pi(x)=|f(x)| / \sum_{x^{\prime} \in \mathscr{X}}\left|f\left(x^{\prime}\right)\right|$, получаем

$$
\|\Phi\|_{1 \rightarrow p}=\max _{\pi \in \mathscr{P}(\mathscr{X})}\|\Phi \pi\|_{p}
$$

где

$$
\mathscr{P}(\mathscr{X})=\left\{\pi: \pi(x) \geqslant 0, \sum_{x \in \mathscr{X}} \pi(x)=1\right\}
$$


- симплекс всех распределений вероятностей $\pi$ на $\mathscr{X}$. Функция $\pi \rightarrow\|\Phi \pi\|_{p}$ является непрерывной и выпуклой и поэтому достигает максимума в крайней точке $\mathscr{P}(\mathscr{X})$, т.е. на вырожденном распределении $\delta_{x}$. Поэтому

$$
\|\Phi\|_{1 \rightarrow p}=\max _{x}\left(\sum_{y \in \mathscr{Y}} \Phi(x, y)^{p}\right)^{1 / p} .
$$

Мультипликативность норм

$$
\left\|\Phi_{1} \otimes \Phi_{2}\right\|_{1 \rightarrow p}=\left\|\Phi_{1}\right\|_{1 \rightarrow p}\left\|\Phi_{2}\right\|_{1 \rightarrow p}
$$

тогда непосредственно следует из того, что $\max _{\pi \in \mathscr{P}}\left(\mathscr{X}_{1} \times \mathscr{X}_{2}\right)\left\|\Phi_{1} \otimes \Phi_{2}\right\|_{1 \rightarrow p}$ достигается на некотором вырожденном распределении $\delta_{x_{1} x_{2}}=\delta_{x_{1}} \times \delta_{x_{2}}$ и поэтому равен произведению $\left\|\Phi_{1}\right\|_{1 \rightarrow p}\left\|\Phi_{2}\right\|_{1 \rightarrow p}$. Подчеркнем, что в основе лежит тот факт, что всякая крайняя точка $\mathscr{P}\left(\mathscr{X}_{1} \times \mathscr{X}_{2}\right)$ является произведением крайних точек из $\mathscr{P}\left(\mathscr{X}_{j}\right)$ :

$$
\operatorname{ext} \mathscr{P}\left(\mathscr{X}_{1} \times \mathscr{X}_{2}\right)=\operatorname{ext} \mathscr{P}\left(\mathscr{X}_{1}\right) \times \operatorname{ext} \mathscr{P}\left(\mathscr{X}_{2}\right) .
$$

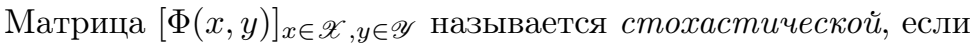

1) $\Phi(x, y) \geqslant 0$

2) $\sum_{y \in \mathscr{Y}} \Phi(x, y)=1$ для всех $x \in \mathscr{X}$.

В теории информации отображение $\Phi$, задаваемое стохастической матрицей, описывает канал (с шумом) из $\mathscr{X}$ в $\mathscr{Y}$. Оно преобразует входное распределение вероятностей $\pi$ на $\mathscr{X}$ в выходное распределение вероятностей $\pi^{\prime}=\Phi \pi$ на $\mathscr{Y}$. Соотношение (5) может быть выражено в терминах аддитивности минимальной выходной энтропии Реньи. Энтропия Реньи порядка $p \geqslant 0$ распределения вероятностей $\pi^{\prime}$ определяется как

$$
R_{p}\left(\pi^{\prime}\right)=\frac{1}{1-p} \log \sum_{y} \pi^{\prime}(y)^{p},
$$

так что минимальная выходная энтропия Реньи канала $\Phi$ равна

$$
\check{R}_{p}(\Phi)=\min _{\pi \in \mathscr{P}(\mathscr{X})} R_{p}(\Phi \pi)=\frac{1}{1-p} \log \|\Phi\|_{1 \rightarrow p}^{p} .
$$

Тогда соотношение (5) принимает вид свойства аддитивности

$$
\check{R}_{p}\left(\Phi_{1} \otimes \Phi_{2}\right)=\check{R}_{p}\left(\Phi_{1}\right)+\check{R}_{p}\left(\Phi_{2}\right) .
$$

В пределе $p \downarrow 1$ энтропии Реньи, монотонно возрастая (и, следовательно, равномерно), сходятся к энтропии Больцмана-Шеннона распределения вероятностей $\pi^{\prime}$ :

$$
\lim _{p \downarrow 1} R_{p}\left(\pi^{\prime}\right)=-\sum_{y} \pi^{\prime}(y) \log \pi^{\prime}(y) \equiv H\left(\pi^{\prime}\right),
$$

так что, вводя минимальную выходную энтропию канала $\Phi$

$$
\check{H}(\Phi)=\min _{\pi \in \mathscr{P}(\mathscr{X})} H(\Phi \pi),
$$


получаем свойство аддитивности

$$
\check{H}\left(\Phi_{1} \otimes \Phi_{2}\right)=\check{H}\left(\Phi_{1}\right)+\check{H}\left(\Phi_{2}\right) .
$$

Минимальные выходные энтропии характеризуют минимальное количество "шума", присутствующего в выходе канала $\Phi$, а свойства (7), (8) показывают, что эти характеристики аддитивны по отношению к независимому использованию каналов. Однако наиболее важной характеристикой такого рода является пропускная способность

$$
C(\Phi)=\max _{\pi \in \mathscr{P}(\mathscr{X})}\left\{H(\Phi \pi)-\sum_{x} \pi(x) H\left(\Phi \delta_{x}\right)\right\},
$$

где выражение в фигурных скобках есть шенноновская взаимная информация между входом и выходом канала. Один из основных результатов теории информации - теорема кодирования для каналов без памяти - утверждает, что величина (9) равна предельной скорости асимптотически безошибочной передачи информации с помощью $n$ независимых использований канала $\Phi$, когда $n \rightarrow \infty$. Пропускная способность имеет аналогичное свойство аддитивности

$$
C\left(\Phi_{1} \otimes \Phi_{2}\right)=C\left(\Phi_{1}\right)+C\left(\Phi_{2}\right)
$$

Здесь неравенство $\geqslant$ (супераддитивность) получается, если ограничиться независимыми входами каналов, тогда как противоположное неравенство может быть доказано с использованием субаддитивности выходной энтропии $H(\Phi \pi)$ и свойства (6) во втором члене шенноновской информации (равном условной выходной энтропии). Это свойство аддитивности является важным ингредиентом доказательства теоремы кодирования, так как оно влечет

$$
C\left(\Phi^{\otimes n}\right)=n C(\Phi)
$$

где $\Phi^{\otimes n}=\Phi \otimes \cdots \otimes \Phi$ ( $n$ раз), однако оно представляется столь “естественным", что обычно не подчеркивается специально.

Далее мы опишем некоммутативные аналоги всех этих понятий, играющих важную роль в квантовой теории информации. Имеется предположение, что соответствующие свойства аддитивности выполняются и в некоммутативном случае, однако до сих пор нет ни общего доказательства, ни опровергающего примера; более того, аддитивность уже не является "естественной", поскольку аналог основного свойства (6) не выполняется в некоммутативном случае. Свойство мультипликативности $p$-норм доказано в ряде случаев, но есть и контрпример, показывающий, что оно может не выполняться для достаточно больших $p$. Это, однако, не исключает возможности его выполнения для $p$, близких к 1, что влекло бы вышеупомянутые свойства аддитивности.

\section{3. Некоммутативный случай}

3.1. Гипотеза мультипликативности. Пусть $\mathscr{H}$ - унитарное пространство и $\mathfrak{M}(\mathscr{H})$ - алгебра всех линейных операторов в $\mathscr{H}$. Фиксируя ортонормированный базис, $\mathscr{H}$ можно отождествить с пространством $\mathscr{H}_{n}$ всех $n$-мерных 
комплексных векторов, а $\mathfrak{M}(\mathscr{H})$ - с алгеброй $\mathfrak{M}_{n}$ всех комплексных $(n \times n)$ матриц. Для $p \geqslant 1$ норма Шаттена [8] оператора $F \in \mathfrak{M}(\mathscr{H})$ определяется как

$$
\|F\|_{p}=\left(\operatorname{Tr}|F|^{p}\right)^{1 / p},
$$

где $\operatorname{Tr}-$ след в $\mathfrak{M}(\mathscr{H})$, а $|F|=\sqrt{F^{*} F}-$ модуль оператора $F$. Случай $p=\infty$ охватывается, если положить $\|F\|_{\infty}$ равным операторной норме $F$, но мы не будем каждый раз оговаривать это явным образом.

Если матрица $F$ эрмитова, то $F=U f U^{*}$, где $U$ - унитарная матрица, $f=\operatorname{diag}(f(x))$ - диагональная матрица с элементами $f(x)$, и $\|F\|_{p}=\|f\|_{p}$ классическая $l^{p}$-норма последовательности $f=(f(x))$. Однако для семейства некоммутирующих матриц $F$ унитарные матрицы $U$ не могут быть одинаковыми. Это приводит к существенному отличию от классического пространства $l^{p}$, которое с этой точки зрения может рассматриваться как состоящее из диагональных (либо одновременно диагонализуемых) матриц, действующих в унитарном пространстве $l^{2}$.

Мы будем рассматривать линейные отображения $\Phi$, которые переводят операторы $(d \times d$-матрицы) $F$ в $d$-мерном унитарном пространстве $\mathscr{H}$ в операторы $\left(d^{\prime} \times d^{\prime}\right.$-матрицы) $F^{\prime}=\Phi(F)$ в $d^{\prime}$-мерном $\mathscr{H}^{\prime}$. Иногда такие отображения называются "супероператорами" или "суперматрицами", поскольку могут быть описаны матрицами с $d^{2} \times d^{2}$ элементами [9]. Рассмотрим нормы таких отображений, определяемые соотношением

$$
\|\Phi\|_{q \rightarrow p}=\max _{F \neq 0} \frac{\|\Phi(F)\|_{p}}{\|F\|_{q}} .
$$

Пусть $\Phi_{j}: \mathfrak{M}\left(\mathscr{H}_{j}\right) \rightarrow \mathfrak{M}\left(\mathscr{H}_{j}^{\prime}\right) ; j=1,2,-$ два таких отображения. Реализуем пространство $\mathscr{H}_{j}$ как $\mathscr{H}_{d_{j}}$, а алгебры $\mathfrak{M}\left(\mathscr{H}_{j}\right)$ как $\mathfrak{M}_{d_{j}}$. Теперь рассмотрим тензорное произведение унитарных пространств $\mathscr{H}_{d_{1}} \otimes \mathscr{H}_{d_{2}}$, которое изоморфно $\mathscr{H}_{d_{1} d_{2}}$ [10]. Изоморфизм может быть установлен путем выбора ортонормированных базисов $\left\{e_{j 1}\right\},\left\{e_{k 2}\right\}$ в $\mathscr{H}_{d_{1}}, \mathscr{H}_{d_{2}}$ и объявления формальных произведений $e_{j 1} \otimes e_{k 2}$ элементами ортонормированного базиса в $\mathscr{H}_{d_{1} d_{2}}$. Поэтому отображение $\Phi_{1} \otimes \Phi_{2}: \mathfrak{M}\left(\mathscr{H}_{1} \otimes \mathscr{H}_{2}\right) \rightarrow \mathfrak{M}\left(\mathscr{H}_{1}^{\prime} \otimes \mathscr{H}_{2}^{\prime}\right)$, определенное естественным образом на произведениях и затем продолженное по линейности, может рассматриваться как отображение из $\mathfrak{M}_{d_{1} d_{2}}$ в $\mathfrak{M}_{d_{1}^{\prime} d_{2}^{\prime}}$, и, таким образом, его $q \rightarrow p$ нормы могут быть однозначно определены. Тогда общая гипотеза мультипликативности [11], [12] формулируется как

$$
\left\|\Phi_{1} \otimes \Phi_{2}\right\|_{q \rightarrow p} \stackrel{?}{=}\left\|\Phi_{1}\right\|_{q \rightarrow p}\left\|\Phi_{2}\right\|_{q \rightarrow p}
$$

для некоторых $q, p \geqslant 1$ и некоторых классов отображений $\Phi_{1}, \Phi_{2}$. Отметим, что вновь неравенство $\geqslant$ легко вытекает из определения нормы, путем сужения максимизации в левой части на векторы-произведения.

Хотя имеется некоммутативный аналог неравенства Минковского [13], это не решает проблему, поскольку доказательство в коммутативном случае существенно опирается на поточечные оценки норм, не имеющие некоммутативного аналога. Основываясь на далеко продвинутой теории операторных $L_{p}$-пространств [14], можно получить интересный результат, касающийся мультипликативности вполне ограниченных p-норм, который, однако, приводит к аддитивности совершенно другой энтропийной характеристики, интерпретация и применимость которой пока не ясны [15]. 
Оператор $F \in \mathfrak{M}(\mathscr{H})$ называется положительным, $F \geqslant 0$, если соответствующая матрица неотрицательно определена, и отображение $\Phi: \mathfrak{M}(\mathscr{H}) \rightarrow$ $\mathfrak{M}\left(\mathscr{H}^{\prime}\right)$ называется положительным, если $F \geqslant 0$ влечет $\Phi(F) \geqslant 0$. Положительное отображение с необходимостью является эрмитовым, т.е. $\Phi\left(F^{*}\right)=$ $\Phi(F)^{*}$ для всех $F$.

Особенно важным будет для нас класс вполне положительных (в.п.) отображений [16], [9]. Отображение $\Phi: \mathfrak{M}(\mathscr{H}) \rightarrow \mathfrak{M}\left(\mathscr{H}^{\prime}\right)$ называется вполне положительным, если для $d=1,2, \ldots$ отображения $\Phi \otimes \operatorname{Id}_{d}$ положительны, где $\mathrm{Id}_{d}: \mathfrak{M}_{d} \rightarrow \mathfrak{M}_{d}$ обозначает тождественное отображение $d \times d$-матриц. Отсюда вытекает, что тензорное произведение в.п. отображений также является в.п. отображением, поскольку

$$
\Phi_{1} \otimes \Phi_{2}=\left(\operatorname{Id}_{d_{1}^{\prime}} \otimes \Phi_{2}\right) \circ\left(\Phi_{1} \otimes \operatorname{Id}_{d_{2}}\right) .
$$

Имеются положительные отображения, которые не являются в.п., например, транспонирование матриц $F \rightarrow F^{T}$ в фиксированном базисе.

Хотя гипотеза мультипликативности для случаев, когда $q \neq 1$ и хотя бы одно из отображений $\Phi_{1}, \Phi_{2}$ не в.п., рассматривалась в литературе (см. [17], [18]), нас будет интересовать специальный случай в.п. отображений с $p$-нормами $\|\Phi\|_{1 \rightarrow p}$, который представляет основной интерес в связи с проблемами аддитивности в квантовой теории информации.

Конечные квантовые системы описываются унитарными пространствами $\mathscr{H}$. Выпуклое подмножество алгебры $\mathfrak{M}(\mathscr{H})$

$$
\mathfrak{S}(\mathscr{H})=\{\rho: \rho \geqslant 0, \operatorname{Tr} \rho=1\}
$$

называется пространством квантовых состояний. Эрмитовы операторы $\rho$ из $\mathfrak{S}(\mathscr{H})$ называются операторами плотности или квантовыми состояниями. Пространство состояний является выпуклым множеством с подмножеством крайних точек

$$
\mathfrak{P}(\mathscr{H})=\operatorname{ext} \mathfrak{S}(\mathscr{H})=\left\{\rho: \rho \geqslant 0, \operatorname{Tr} \rho=1, \rho^{2}=\rho\right\} .
$$

Таким образом, крайние точки множества $\mathfrak{S}(\mathscr{H})$, которые также называются чистыми состояниями, суть одномерные проекторы $\rho=P_{\psi}$ на векторы $\psi \in \mathscr{H}$ с единичной нормой (см., например, [1]). Подробнее о геометрии пространства квантовых состояний см. в [19].

В.п. отображение $\Phi$ называется каналом, если оно сохраняет след, т.е. переводит состояния в состояния (возможно, в другом унитарном пространстве $\mathscr{H}^{\prime}$ ). Канал $\Phi$ называется унитальным, если $d_{B}=d_{A}$ и $\Phi\left(I_{A}\right)=I_{B}$.

3.2. Пример: унитальные кубитные каналы. Простейший и в то же время важный пример дает кубит (квантовый бит) - двумерная квантовая система, $\operatorname{dim} \mathscr{H}=2$. Удобный линейный базис в $\mathfrak{M}_{2}$ образуют матрицы Паули

$$
I \equiv \sigma_{0}=\left[\begin{array}{ll}
1 & 0 \\
0 & 1
\end{array}\right], \quad \sigma_{x}=\left[\begin{array}{ll}
0 & 1 \\
1 & 0
\end{array}\right], \quad \sigma_{y}=\left[\begin{array}{cc}
0 & -i \\
i & 0
\end{array}\right], \quad \sigma_{z}=\left[\begin{array}{cc}
1 & 0 \\
0 & -1
\end{array}\right] .
$$

Они образуют базис и в вещественном линейном пространстве всех эрмитовых $2 \times 2$-матриц. В частности, любой оператор плотности $\rho \in \mathfrak{S}\left(\mathscr{H}_{2}\right)$ может быть представлен матрицей

$$
\rho=\frac{1}{2}\left(I+a_{x} \sigma_{x}+a_{y} \sigma_{y}+a_{z} \sigma_{z}\right)=\frac{1}{2}\left[\begin{array}{cc}
1+a_{z} & a_{x}-i a_{y} \\
a_{x}+i a_{y} & 1-a_{z}
\end{array}\right],
$$


где параметры Стокса $\vec{a}=\left(a_{x}, a_{y}, a_{z}\right)$ удовлетворяют условию

$$
|\vec{a}|^{2} \equiv a_{x}^{2}+a_{y}^{2}+a_{z}^{2} \leqslant 1 .
$$

Таким образом, множество состояний $\mathfrak{S}\left(\mathscr{H}_{2}\right)$ как выпуклое множество изоморфно единичному шару в $\mathbb{R}^{3}$. Собственные значения оператора плотности (14) суть $(1 \pm|\vec{a}|) / 2$.

Чистые состояния характеризуются условием $a_{x}^{2}+a_{y}^{2}+a_{z}^{2}=1$ и образуют сферу $\mathfrak{P}\left(\mathscr{H}_{2}\right)$. Вводя углы Эйлера $\theta, \phi$, так что $a_{z}=\cos \theta, a_{x}+i a_{y}=\sin \theta e^{i \phi}$, имеем $\rho=P_{\psi(\vec{a})}$, где

$$
\psi(\vec{a})=\left[\begin{array}{c}
\cos \frac{\theta}{2} e^{-i \phi / 2} \\
\sin \frac{\theta}{2} e^{i \phi / 2}
\end{array}\right] .
$$

ПримечАНиЕ. Примером физического кубита является атом с двумя выделенными энергетическими уровнями.

Другой пример - спин электрона. Вектор $\psi(\vec{a})$ описывает чистое состояние с направлением спина $\vec{a}$. Состояние с $a_{x}=a_{y}=a_{z}=0$, описываемое оператором плотности $\rho=\frac{1}{2} I$, для которого все направления спина равновероятны, называется хаотическим.

Еще один важный пример дается поляризацией монохроматического фотона, визуализуемой в классических оптических экспериментах. В этом случае параметр $\theta / 2$ задает угол линейной поляризации, а $\phi / 2$ характеризует круговую поляризацию.

Рассмотрим подробнее структуру кубитных в.п. отображений и каналов.

ПреДложениЕ 1 (см., например, [20]). Произволъное линейное эрмитово отображение $\Phi: \mathfrak{M}_{2} \rightarrow \mathfrak{M}_{2}$, сохраняющее след, может быть представлено в виде

$$
\Phi(\rho)=U_{2} \Lambda\left(U_{1} \rho U_{1}^{*}\right) U_{2}^{*},
$$

где $U_{1}, U_{2}$ - унитарные матрицы, а отображение $\Lambda$ имеет следующую каноническую форму в базисе матрии, Паули:

$$
\Lambda(I)=I+\sum_{\gamma=x, y, z} t_{\gamma} \sigma_{\gamma}, \quad \Lambda\left(\sigma_{\gamma}\right)=\lambda_{\gamma} \sigma_{\gamma}, \quad \gamma=x, y, z,
$$

где $\lambda_{\gamma}, t_{\gamma}$ вещественны. Представление (17) всегда может быть выбрано таким образом, что не более чем одна из величин $\lambda_{\gamma}$ является отрицательной.

ДокАзАтельство. Отображение $\Phi$ аффинно на $\mathfrak{S}\left(\mathscr{H}_{2}\right)$, поэтому оно отображает состояние $\rho(\vec{a})$, задаваемое формулой (14), в эрмитов оператор $\rho(T \vec{a}+\vec{b})$ с единичным следом, где $T$ - вещественная $(3 \times 3)$-матрица. Используя полярное разложение матрицы $T$, а затем спектральное представление $|T|$, получаем

$$
T=O|T|=O_{2} L O_{1}
$$

где $O, O_{1}, O_{2}-$ ортогональные матрицы, $\operatorname{det} O_{1}=\operatorname{det} O_{2}=1$ и $L=\operatorname{diag}\left(\lambda_{x}, \lambda_{y}, \lambda_{z}\right)$ - диагональная матрица с вещественными $\lambda$. Таким образом,

$$
\Phi(\rho(\vec{a}))=\rho\left(O_{2}\left(L\left(O_{1} \vec{a}\right)+\vec{t}\right)\right)
$$

где $\vec{t}=O_{2}^{-1} \vec{b}$. 
Матрицы $O_{1}, O_{2}$ задают вращения в $\mathbb{R}^{3}$ и представляются унитарными операторами $U_{1}, U_{2}$ в $\mathscr{H}$. Вместе с (18) это завершает доказательство формулы (16).

Числа $\lambda_{x}, \lambda_{y}, \lambda_{z}$ являются собственными значениями оператора $|T|$ либо оператора $-|T|$, поэтому либо все неотрицательны, либо все неположительны. В последнем случае мы можем умножить $L$ на диагональную матрицу $\operatorname{diag}(-1,-1,1)$, которая отвечает вращению вокруг оси $z$ на угол $\pi$ и поэтому может быть компенсирована надлежащим выбором матрицы $O_{1}$. Это доказывает утверждение о знаках. Предложение 1 доказано.

Полученное представление означает, что, с точностью до несущественных для дальнейшего унитарных преобразований, произвольное эрмитово кубитное отображение задается соотношениями (17). Это означает, что состояние (14) преобразуется в эрмитов оператор $\rho^{\prime}$ с параметрами

$$
a_{x}^{\prime}=\lambda_{x} a_{x}+t_{x}, \quad a_{y}^{\prime}=\lambda_{y} a_{y}+t_{y}, \quad a_{z}^{\prime}=\lambda_{z} a_{z}+t_{z},
$$

поэтому единичный шар как целое преобразуется в эллипсоид с центром $\left(t_{x}, t_{y}, t_{z}\right)$ и главными осями $x, y, z$ с длинами полуосей $\left|\lambda_{x}\right|,\left|\lambda_{y}\right|,\left|\lambda_{z}\right|$ соответственно. Разумеется, полная положительность налагает нетривиальные ограничения на параметры $\lambda_{\gamma}, t_{\gamma}$, см., например [20]. Эти ограничения приобретают особенно прозрачную форму в случае $t_{\gamma} \equiv 0$, когда отображение сжимает единичный шар вдоль осей $x, y, z$ с коэффициентами $\left|\lambda_{x}\right|,\left|\lambda_{y}\right|,\left|\lambda_{z}\right|$. Это имеет место тогда и только тогда, когда отображение $\Phi$, а следовательно, и $\Lambda$ унитально.

В этом случае, используя правила умножения для матриц Паули, получаем

$$
\Lambda(\rho)=\sum_{\gamma=0, x, y, z} \mu_{\gamma} \sigma_{\gamma} \rho \sigma_{\gamma},
$$

где

$$
\begin{array}{ll}
\mu_{0}=\frac{1}{4}\left(1+\lambda_{x}+\lambda_{y}+\lambda_{z}\right), \quad \mu_{x}=\frac{1}{4}\left(1+\lambda_{x}-\lambda_{y}-\lambda_{z}\right), \\
\mu_{y}=\frac{1}{4}\left(1-\lambda_{x}+\lambda_{y}-\lambda_{z}\right), \quad \mu_{z}=\frac{1}{4}\left(1-\lambda_{x}-\lambda_{y}+\lambda_{z}\right),
\end{array}
$$

и неотрицательность этих чисел является необходимым и достаточным условием для полной положительности отображения $\Lambda$, а следовательно, и $\Phi$. Это видно из того, что соотношение (19) есть не что иное, как представление (44) для данного частного случая.

Геометрия пространства состояний и действие в.п. отображений в размерностях $d>2$ не допускают столь же наглядного описания. Имеется, однако, некоторое обобщение представления состояний (14) и каналов типа (19), использующее дискретную версию операторов Вейля (вместо матриц Паули) и соответствующее некоммутативное преобразование Фурье [21].

3.3. Энтропийные характеристики в.п. отображений и каналов. Как показано в работах [22], [23] (см. приложение), в случае в.п. отображения $\Phi$ выполняется равенство

$$
\|\Phi\|_{1 \rightarrow p}=\max _{\rho \in \mathfrak{S}(\mathscr{H})}\left[\operatorname{Tr} \Phi(\rho)^{p}\right]^{1 / p} \equiv \nu_{p}(\Phi),
$$


где в правой части стоит характеристика "чистоты выхода" канала $\Phi$, введенная в [11]. Она соответствует величине (4) в некоммутативном случае ${ }^{1}$. Однако, в отличие от коммутативного случая, нет очевидной причины, в силу которой должна была бы выполняться мультипликативность

$$
\nu_{p}\left(\Phi_{1} \otimes \Phi_{2}\right) \stackrel{?}{=} \nu_{p}\left(\Phi_{1}\right) \nu_{p}\left(\Phi_{2}\right)
$$

так как аналог свойства (6) уже не имеет места. На самом деле

$$
\operatorname{ext} \mathfrak{S}\left(\mathscr{H}_{1} \otimes \mathscr{H}_{2}\right) \supsetneqq \operatorname{ext} \mathfrak{S}\left(\mathscr{H}_{1}\right) \times \operatorname{ext} \mathfrak{S}\left(\mathscr{H}_{2}\right)
$$

поскольку, очевидно, имеется континуально много чистых состояний $P_{\psi}$ в $\mathscr{H}_{1} \otimes \mathscr{H}_{2}$, задаваемых векторами $\psi$, которые не представимы в виде тензорного произведения $\psi_{1} \otimes \psi_{2}$ (а именно, все линейные комбинации таких векторов, не сводящиеся к произведению).

ПримечАниЕ. В квантовой теории тензорное произведение $\mathscr{H}_{1} \otimes \mathscr{H}_{2}$ описывает составную (двухчастичную) систему. Векторы, не представимые в виде $\psi_{1} \otimes \psi_{2}$, как и соответствующие чистые состояния, называются сцепленными. Для сцепленного чистого состояния двухчастичной квантовой системы ни одна из ее частей не находится в чистом состоянии, что резко отличает квантовые системы от классических.

Тем не менее мультипликативность (21) может быть доказана при всех $p \geqslant 1$ для целого ряда случаев, включающих и все унитальные кубитные каналы, хотя имеется пример, где она нарушается для достаточно больших $p$. Это будет рассмотрено в разделе 5. Как будет показано ниже, выполнимость гипотезы мультипликативности для всех каналов и $p \in[1,1+\varepsilon)$, где $\varepsilon>0$, влечет выполнимость всех важнейших свойств аддитивности в квантовой теории информации. На настоящий момент правдоподобная гипотеза состоит в том, что (21) выполняется для всех в.п. отображений по крайней мере при $p \in[1,2]$.

Аналогично коммутативному случаю, соотношение (21) может быть переформулировано как аддитивность минимальной выходной энтропии Реньи. Квантовая энтропия Реньи порядка $p \geqslant 0$ оператора плотности $\rho$ равна

$$
R_{p}(\rho)=\frac{1}{1-p} \log \operatorname{Tr} \rho^{p}
$$

так что минимальная выходная энтропия Реньи канала $\Phi$ есть

$$
\check{R}_{p}(\Phi)=\min _{\rho \in \mathfrak{S}(\mathscr{H})} R_{p}(\Phi(\rho))=\frac{p}{1-p} \log \nu_{p}(\Phi)
$$

Соотношение (21) принимает форму

$$
\check{R}_{p}\left(\Phi_{1} \otimes \Phi_{2}\right) \stackrel{?}{=} \check{R}_{p}\left(\Phi_{1}\right)+\check{R}_{p}\left(\Phi_{2}\right)
$$

\footnotetext{
${ }^{1} \mathrm{~B}$ конечномерном случае легко доказывается, что все рассматриваемые нами функции состояний (такие, как определяемые далее энтропии) непрерывны и достигают своих экстремумов на множестве состояний. Однако в бесконечномерном случае это уже не так, и достижимость экстремумов требует специального рассмотрения, см. раздел 7.
} 
В пределе $p \downarrow 1$ квантовые энтропии Реньи монотонно возрастают и стремятся к энтропии фон Неймана оператора плотности $\rho$

$$
\lim _{p \downarrow 1} R_{p}(\rho)=-\operatorname{Tr} \rho \log \rho \equiv H(\rho),
$$

так что, вводя минимальную выходную энтропию квантового канала $\Phi$

$$
\check{H}(\Phi)=\min _{\rho \in \mathfrak{S}(\mathscr{H})} H(\Phi(\rho)),
$$

получаем свойство аддитивности

$$
\check{H}\left(\Phi_{1} \otimes \Phi_{2}\right) \stackrel{?}{=} \check{H}\left(\Phi_{1}\right)+\check{H}\left(\Phi_{2}\right),
$$

при условии, что гипотеза мультипликативности (21) выполняется для $p \in$ $[1,1+\varepsilon)$. Вновь неравенство $\leqslant$ является здесь очевидным.

Некоммутативным аналогом величины (9) является $\chi$-пропускная способность

$$
C_{\chi}(\Phi)=\max _{\pi}\left\{H\left(\Phi\left(\sum_{x} \pi(x) \rho(x)\right)\right)-\sum_{x} \pi(x) H(\Phi(\rho(x)))\right\},
$$

где максимум ${ }^{2}$ берется по всевозможным ансамблям состояний, т.е. конечным распределениям вероятностей $\pi$ на множестве квантовых состояний $\mathfrak{S}(\mathscr{H})$, приписывающим вероятности $\pi(x)$ операторам плотности $\rho(x)$. Соответствующая гипотеза аддитивности состоит в выполнимости аналога свойства (10) для квантовых каналов, т.е.

$$
C_{\chi}\left(\Phi_{1} \otimes \Phi_{2}\right) \stackrel{?}{=} C_{\chi}\left(\Phi_{1}\right)+C_{\chi}\left(\Phi_{2}\right) .
$$

Здесь вновь $\otimes$ обозначает тензорное произведение каналов, описывающее их независимое применение к состояниям составной системы, и неравенство $\geqslant$ является очевидным. Это первая из проблем аддитивности в квантовой теории информации, формулировка которой восходит к работе [25], см. также [26], [2].

ПримечАниЕ. В физических терминах эта проблема может быть переформулирована так: "Может ли сцепленность состояний сигнала на входе способствовать передаче классической информации через квантовый канал связи?" Напомним, что пропускная способность канала определяется как максимальная скорость передачи, с произвольными кодированием и декодированием для возрастающего числа $n$ независимых использований канала

$$
\Phi^{\otimes n}=\underbrace{\Phi \otimes \cdots \otimes \Phi}_{n},
$$

при которых вероятность ошибки стремится к нулю при $n \rightarrow \infty$. Один из основных результатов квантовой теории информации утверждает, что так определенная пропускная способность ${ }^{3}$ дается формулой

$$
C(\Phi)=\lim _{n \rightarrow \infty} \frac{1}{n} C_{\chi}\left(\Phi^{\otimes n}\right) .
$$

\footnotetext{
${ }^{2}$ В рассматриваемом нами конечномерном случае максимум достигается на $\pi$ с носителем, состоящим из не более чем $d^{2}$ состояний, где $d=\operatorname{dim} \mathscr{H}[24]$.

${ }^{3}$ Она называется классической пропускной способностъю квантового канала $\Phi$ [26], [1]. Квантовый канал характеризуется целым набором пропускных способностей, зависящих от вида передаваемой информации (классической или квантовой), ограничений на процедуры кодирования и декодирования и от возможных дополнительных ресурсов, используемых при передаче [2].
} 
Поскольку величина $C_{\chi}(\Phi)$, как легко видеть, является супераддитивной (т.е. $\left.C_{\chi}\left(\Phi_{1} \otimes \Phi_{2}\right) \geqslant C_{\chi}\left(\Phi_{1}\right)+C_{\chi}\left(\Phi_{2}\right)\right)$, имеет место неравенство $C(\Phi) \geqslant C_{\chi}(\Phi)$. Если же выполняется аддитивность $(27)$, то $C_{\chi}\left(\Phi^{\otimes n}\right)=n C_{\chi}(\Phi)$, и это неравенство превращается в равенство. Такой результат был бы весьма желательным с математической точки зрения, поскольку давал бы сравнительно легко вычислимое "однобуквенное" выражение для классической пропускной способности квантового канала.

3.4. Различные формы гипотезы аддитивности. Формулировка в терминах двойственности выпуклого анализа. Из определения $\chi$-пропускной способности вытекает, что

$$
C_{\chi}(\Phi) \leqslant \max _{\rho \in \mathfrak{S}(\mathscr{H})} H(\Phi(\rho))-\min _{\rho \in \mathfrak{S}(\mathscr{H})} H(\Phi(\rho)) .
$$

Чтобы полнее раскрыть связь между выходной энтропией и $\chi$-пропускной способностью, перепишем выражение (26) в виде

$$
C_{\chi}(\Phi)=\max _{\rho \in \mathfrak{S}(\mathscr{H})}\left[H(\Phi(\rho))-\hat{H}_{\Phi}(\rho)\right]
$$

где

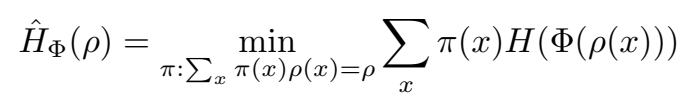

есть выпуклое замыкание [27] выходной энтропии $H(\Phi(\rho))^{4}$. Здесь минимум берется по всевозможным конечным распределениям вероятностей на $\mathfrak{S}(\mathscr{H})$. Гипотетическое свойство супераддитивности этой функции гласит: для любого состояния $\rho_{12} \in \mathfrak{S}\left(\mathscr{H}_{1} \otimes \mathscr{H}_{2}\right)$ и произвольных каналов $\Phi_{1} \otimes \Phi_{2}$

$$
\hat{H}_{\Phi_{1} \otimes \Phi_{2}}\left(\rho_{12}\right) \stackrel{?}{\geqslant} \hat{H}_{\Phi_{1}}\left(\rho_{1}\right)+\hat{H}_{\Phi_{2}}\left(\rho_{2}\right),
$$

где $\rho_{1}, \rho_{2}$ - частичные следы состояния $\rho_{12}$ в $\mathscr{H}_{1}, \mathscr{H}_{2}$ (относительно понятия частичного следа см. следующий раздел).

ПРЕДЛОЖЕНИЕ 2. Свойство супераддитивности (30) влечет свойства аддитивности (25) и (27) для данных каналов $\Phi_{1}, \Phi_{2}$.

ДокАЗАТЕЛЬство. В самом деле, пусть $\rho_{12}^{0}$ минимизирует функцию $H\left(\left(\Phi_{1} \otimes \Phi_{2}\right)\left(\rho_{12}\right)\right)$, тогда

$$
\begin{aligned}
\check{H}\left(\Phi_{1} \otimes \Phi_{2}\right) & =H\left(\left(\Phi_{1} \otimes \Phi_{2}\right)\left(\rho_{12}^{0}\right)\right) \geqslant \hat{H}_{\Phi_{1} \otimes \Phi_{2}}\left(\rho_{12}^{0}\right) \\
& \geqslant \hat{H}_{\Phi_{1}}\left(\rho_{1}^{0}\right)+\hat{H}_{\Phi_{2}}\left(\rho_{2}^{0}\right) \geqslant \check{H}\left(\Phi_{1}\right)+\check{H}\left(\Phi_{2}\right),
\end{aligned}
$$

откуда следует (25). С другой стороны, свойство (30) и субаддитивность квантовой энтропии

$$
H\left(\sigma_{12}\right) \leqslant H\left(\sigma_{1}\right)+H\left(\sigma_{2}\right)
$$

\footnotetext{
43десь применимо такое же замечание о достижимости максимума, что и в случае (26). Функция $\hat{H}_{\Phi}(\rho)$ является естественным обобщением другого важного понятия в квантовой теории информации - "сцепленность формирования" (entanglement of formation) состояния $\rho[2]$, и сводится к нему, если канал $\Phi$ представляет собой частичный след.
} 
(см., например, [1]) влекут

$$
\begin{aligned}
H\left(\left(\Phi_{1} \otimes \Phi_{2}\right)\left(\rho_{12}\right)\right)-\hat{H}_{\Phi_{1} \otimes \Phi_{2}}\left(\rho_{12}\right) \\
\quad \leqslant H\left(\left(\Phi_{1} \otimes \Phi_{2}\right)\left(\rho_{12}\right)\right)-\hat{H}_{\Phi_{1}}\left(\rho_{1}\right)-\hat{H}_{\Phi_{2}}\left(\rho_{2}\right) \\
\quad \leqslant\left[H\left(\Phi_{1}\left(\rho_{1}\right)\right)-\hat{H}_{\Phi_{1}}\left(\rho_{1}\right)\right]+\left[H\left(\Phi_{2}\left(\rho_{2}\right)\right)-\hat{H}_{\Phi_{2}}\left(\rho_{2}\right)\right] .
\end{aligned}
$$

Используя (29), получаем

$$
C_{\chi}\left(\Phi_{1} \otimes \Phi_{2}\right) \leqslant C_{\chi}\left(\Phi_{1}\right)+C_{\chi}\left(\Phi_{2}\right)
$$

т.е. (27). Предложение 2 доказано.

Как будет показано в разделе 6, свойства аддитивности (25) и (27) равносильны в специальном случае неприводимо ковариантных каналов $\Phi_{1}, \Phi_{2}$. Замечательно, однако, что все эти свойства оказываются эквивалентными глобально, т.е. если они выполняются для всех каналов.

Теорема 1 [28]. Гипотезы (25), (27) и (30) глобально эквивалентны в том смысле, что если одна из них выполняется для всех каналов $\Phi_{1}, \Phi_{2}$, то любая другая также выполняется для всех каналов.

Доказательство, которое будет дано в разделе 6 , использует еще одну разновидность гипотезы аддитивности. В работе [29] для исследования проблемы мультипликативности/аддитивности были привлечены средства выпуклого анализа. Для данного канала $\Phi$ выходная энтропия $H(\Phi(\rho))$ является непрерывной функцией на множестве состояний $\mathfrak{S}(\mathscr{H})$. Рассмотрим ее преобразование Лежандра

$$
H_{\Phi}^{*}(X)=\max _{\rho \in \mathfrak{S}(\mathscr{H})}\{\operatorname{Tr} \rho X-H(\Phi(\rho))\}, \quad X \in \mathfrak{M}_{h}(\mathscr{H}),
$$

где $\mathfrak{M}_{h}(\mathscr{H})$ - вещественное нормированное пространство эрмитовых операторов в $\mathscr{H}$. Это выпуклая функция на $\mathfrak{M}_{h}(\mathscr{H})$, чье преобразование Лежандра

$$
H_{\Phi}^{* *}(\rho)=\max _{X \in \mathfrak{M}_{h}(\mathscr{H})}\left\{\operatorname{Tr} \rho X-H_{\Phi}^{*}(X)\right\}
$$

совпадает с выпуклым замыканием исходной функции,

$$
H_{\Phi}^{* *}(\rho)=\hat{H}_{\Phi}(\rho),
$$

как следует из теоремы Фенхеля [27]. Далее, преобразование Лежандра функции $\hat{H}_{\Phi}(\rho)$ совпадает с $H_{\Phi}^{*}(X)$, т.е.

$$
H_{\Phi}^{*}(X)=\max _{\rho \in \mathfrak{S}(\mathscr{H})}\left\{\operatorname{Tr} \rho X-\hat{H}_{\Phi}(\rho)\right\} .
$$

Пусть теперь $\Phi_{1}, \Phi_{2}$ - два данных канала.

Лемма 1. Супераддитивность (30) выпуклого замыкания $\hat{H}_{\Phi}(\rho)$ равносильна следующему свойству аддитивности функиии $H_{\Phi}^{*}(X)$ :

$$
H_{\Phi_{1} \otimes \Phi_{2}}^{*}\left(X_{1} \otimes I_{2}+I_{1} \otimes X_{2}\right)=H_{\Phi_{1}}^{*}\left(X_{1}\right)+H_{\Phi_{2}}^{*}\left(X_{2}\right)
$$

для всех $X_{1} \in \mathfrak{M}_{h}\left(\mathscr{H}_{1}\right), X_{2} \in \mathfrak{M}_{h}\left(\mathscr{H}_{2}\right)$. 
ДокАЗАТЕЛЬСтво. В соотношении (34) неравенство $\geqslant$ всегда выполняется, поскольку правая часть получается из левой ограничением $\rho=\rho_{1} \otimes \rho_{2}$ в максимизации (31), определяющей $H_{\Phi_{1} \otimes \Phi_{2}}^{*}$.

Пусть имеет место свойство (30), тогда, обозначая $Y=X_{1} \otimes I_{2}+I_{1} \otimes X_{2}$ и используя (33), получаем

$$
\begin{aligned}
H_{\Phi_{1} \otimes \Phi_{2}}^{*}(Y) & =\max _{\rho_{12}}\left\{\operatorname{Tr} \rho_{12} Y-\hat{H}_{\Phi}\left(\rho_{12}\right)\right\} \\
& \leqslant \max _{\rho_{1}, \rho_{2}}\left\{\operatorname{Tr} \rho_{1} X_{1}+\operatorname{Tr} \rho_{2} X_{2}-\hat{H}_{\Phi}\left(\rho_{1}\right)-\hat{H}_{\Phi}\left(\rho_{2}\right)\right\} \\
& =H_{\Phi_{1}}^{*}\left(X_{1}\right)+H_{\Phi_{2}}^{*}\left(X_{2}\right),
\end{aligned}
$$

поэтому выполняется (34).

Обратное утверждение получается аналогично, применением (32) и равенства $\hat{H}_{\Phi}(\rho)=H_{\Phi}^{* *}(\rho)$. Лемма 1 доказана.

\section{4. Свойства вполне положительных отображений}

4.1. Обозначения Дирака. В дальнейшем изложении мы будем систематически использовать обозначения Дирака, т.е. обозначать векторы $\psi \in \mathscr{H}$ символом $|\psi\rangle$, а соответствующие линейные функционалы на $\mathscr{H}$ - символом $\langle\psi|$. Скалярное произведение в $\mathscr{H}$ тогда обозначается $\langle\varphi \mid \psi\rangle$, и оно линейно по второму аргументу. Оператор $X$ ранга 1 , действующий на вектор $\chi \in \mathscr{H}$ по формуле $X \chi=\psi\langle\varphi \mid \chi\rangle$, будет обозначаться символом $|\psi\rangle\langle\varphi|$ (внешнее произведение). Тогда $P_{\psi}=|\psi\rangle\langle\psi|$ - одномерный проектор на $\psi$, единичный оператор есть

$$
I=\sum_{j=1}^{d}\left|e_{j}\right\rangle\left\langle e_{j}\right|,
$$

где $\left\{e_{j}\right\}$ - какой-либо ортонормированный базис в $\mathscr{H}$, а след оператора равен

$$
\operatorname{Tr} X=\sum_{j=1}^{d}\left\langle e_{j}|X| e_{j}\right\rangle .
$$

Для данного оператора $X: \mathscr{H}_{A} \rightarrow \mathscr{H}_{B} \otimes \mathscr{H}_{C}$ и вектора $\psi_{C} \in \mathscr{H}_{C}$ мы обозначаем $\left\langle\psi_{C}\right| X$ оператор $Y: \mathscr{H}_{A} \rightarrow \mathscr{H}_{B}$ такой, что

$$
\left\langle\psi_{B}|Y| \psi_{A}\right\rangle=\left\langle\psi_{B} \otimes \psi_{C}|X| \psi_{A}\right\rangle \quad \text { для всех } \psi_{A}, \psi_{B}
$$

аналогично, в случае $X: \mathscr{H}_{A} \otimes \mathscr{H}_{C} \rightarrow \mathscr{H}_{B}$ мы можем определить оператор $X\left|\psi_{C}\right\rangle: \mathscr{H}_{A} \rightarrow \mathscr{H}_{B}$ и т. п.

Для данного оператора $V: \mathscr{H}_{A} \rightarrow \mathscr{H}_{B}$ и вектора $e \in \mathscr{H}_{C}$ мы обозначаем $V \otimes|e\rangle$ оператор $X: \mathscr{H}_{A} \rightarrow \mathscr{H}_{B} \otimes \mathscr{H}_{C}$ такой, что

$$
X\left|\psi_{A}\right\rangle=V\left|\psi_{A}\right\rangle \otimes|e\rangle \quad \text { для всех } \psi_{A} ;
$$

аналогично, $V \otimes\langle e|$ обозначает оператор $Y: \mathscr{H}_{A} \otimes \mathscr{H}_{C} \rightarrow \mathscr{H}_{B}$ такой, что

$$
Y\left|\psi_{A} \otimes \psi_{C}\right\rangle=V\left|\psi_{A}\right\rangle\left\langle e \mid \psi_{C}\right\rangle \quad \text { для всех } \psi_{A}, \psi_{C} .
$$


Пусть $X_{12}$ оператор в тензорном произведении $\mathscr{H}_{1} \otimes \mathscr{H}_{2}$, тогда его частичный след $\operatorname{Tr}_{\mathscr{H}_{2}} X_{12}$ относительно пространства $\mathscr{H}_{2}$ определяется как оператор $X_{1}$ в $\mathscr{H}_{1}$, ассоциированный с полуторалинейной формой

$$
\left\langle\varphi\left|X_{1}\right| \psi\right\rangle=\sum_{j}\left\langle\varphi \otimes e_{j}^{2}\left|X_{12}\right| \psi \otimes e_{j}^{2}\right\rangle,
$$

где $\left\{e_{j}^{2}\right\}$ - какой-либо ортонормированный базис в $\mathscr{H}_{2}$ (легко доказывается, что эта величина не зависит от выбора базиса) [10]. Если $\rho_{12}$ - оператор плотности (состояние), то $\rho_{1}$ - также оператор плотности, называемый частичным состоянием.

Заметим, что, используя введенные выше обозначения, мы можем записать (39) в виде, аналогичном (36):

$$
X_{1}=\sum_{j}\left\langle e_{j}^{2}\left|X_{12}\right| e_{j}^{2}\right\rangle
$$

4.2. Очищение состояний. Очень полезным инструментом в квантовой теории информации является следующий простой факт, который мы докажем в несколько более общей, чем обычно (см., например, [1], [3]), форме, для произвольных, не обязательно нормированных векторов.

ПредлОжЕНИЕ 3 (Разложение Шмидта). Пусть $X_{12}=|\psi\rangle\langle\psi|$, где $|\psi\rangle \in$ $\mathscr{H}_{1} \otimes \mathscr{H}_{2}, u X_{1}=\operatorname{Tr}_{\mathscr{H}_{2}} X_{12}, X_{2}=\operatorname{Tr}_{\mathscr{H}_{1}} X_{12}$ - частичные следы оператора $X_{12}$. Тогда $X_{1}$ и $X_{2}$ именот одинаковые ненулевые собственные значения $\lambda_{j}$. Более moго,

$$
|\psi\rangle=\sum_{j} \sqrt{\lambda_{j}}\left|e_{j}^{1}\right\rangle \otimes\left|e_{j}^{2}\right\rangle
$$

где $\left\{e_{j}^{1,2}\right\}$ - соответствующие ортонормированные собственные векторы операторов $X_{1}, X_{2}$.

ДокАЗАтЕЛЬство. Выбирая ортонормированный базис $\left\{e_{j}^{1}\right\}$ из собственных векторов $X_{1}$ в $\mathscr{H}_{1}$, мы можем написать

$$
|\psi\rangle=\sum_{j}\left|e_{j}^{1}\right\rangle \otimes\left|h_{j}^{2}\right\rangle
$$

для некоторых $\left|h_{j}^{2}\right\rangle \in \mathscr{H}_{2}$. Беря частичный след, получаем

$$
X_{1}=\sum_{j} \lambda_{j}\left|e_{j}^{1}\right\rangle\left\langle e_{j}^{1}\left|=\sum_{j, k}\left\langle h_{j}^{2} \mid h_{k}^{2}\right\rangle\right| e_{k}^{1}\right\rangle\left\langle e_{j}^{1}\right|,
$$

и поэтому $\left\langle h_{j}^{2} \mid h_{k}^{2}\right\rangle=\lambda_{j} \delta_{j k}$. Следовательно, мы можем положить $\left|e_{j}^{2}\right\rangle=\frac{1}{\sqrt{\lambda_{j}}}\left|h_{j}^{2}\right\rangle$ при $\lambda_{j}>0$. В силу симметрии между подсистемами, эти векторы являются собственными для оператора $X_{2}$ с положительными собственными значениями $\lambda_{j}$. Мы можем теперь дополнить эту ортонормированную систему до базиса из собственных векторов в $\mathscr{H}_{2}$. Предложение 3 доказано.

Следующее утверждение является обратным. 
ПРЕДЛОЖЕНИЕ 4 (Очищение состояний). Пусть $X_{1}$ - положительный оператор в $\mathscr{H}_{1}$, тогда найдутся унитарное пространство $\mathscr{H}_{2}$ той же размерности, что и $\mathscr{H}_{1}$, и вектор $|\psi\rangle \in \mathscr{H}_{1} \otimes \mathscr{H}_{2}$ такие, что $X_{1}=\operatorname{Tr}_{\mathscr{H}_{2}}|\psi\rangle\langle\psi|$. Для любого другого вектора $\left|\psi^{\prime}\right\rangle \in \mathscr{H}_{1} \otimes \mathscr{H}_{2}$ с таким же свойством найдется унитарный оператор $U_{2}$ в $\mathscr{H}_{2}$ такой, что $\left|\psi^{\prime}\right\rangle=\left(I_{1} \otimes U_{2}\right)\left|\psi^{\prime}\right\rangle$.

ДокАзАтельство. Диагонализуем оператор $X_{1}$ и выберем вектор $|\psi\rangle$ как в (41), с произвольным ортонормированным базисом $\left\{e_{j}^{2}\right\}$ в унитарном пространстве $\mathscr{H}_{2}$, изоморфном $\mathscr{H}_{1}$. Любой другой такой вектор $\left|\psi^{\prime}\right\rangle$ допускает разложение (41) с другим базисом в $\mathscr{H}_{2}$, а любые два базиса в одном и том же пространстве связаны унитарным преобразованием $U_{2}$. Предложение 4 доказано.

\section{3. Представления вполне положительных отображений.}

ПреДЛОЖеНИЕ 5. Отображение $\Phi: \mathfrak{M}\left(\mathscr{H}_{A}\right) \rightarrow \mathfrak{M}\left(\mathscr{H}_{B}\right)$ является в.n. тогда и только тогда, когда оно представимо в виде

$$
\Phi(\rho)=\sum_{j=1}^{N} V_{j} \rho V_{j}^{*} ; \quad \rho \in \mathfrak{M}\left(\mathscr{H}_{A}\right),
$$

где $N \leqslant d_{A} d_{B}$. Отображение (44) сохраняет след (т.е. является каналом) тогда и только тогда, когда

$$
\sum_{j=1}^{N} V_{j}^{*} V_{j}=I
$$

Формула (44) обычно называется представлением Крауса, который получил эту формулу (для бесконечномерного случая) как следствие общего результата В.Ф. Стайнспринга [16], относящегося к в.п. отображениям произвольных $C^{*}$-алгебр. Независимо и позднее более простое и прямое доказательство для конечномерного случая было получено в работе М. Д. Чоя [9].

ДокАЗАТЕЛЬСтво. Выберем ортонормированный базис $\left\{e_{j}^{A}\right\}$ в $\mathscr{H}_{A}$ и рассмотрим максимально сцепленный вектор

$$
\left|\Omega^{A A}\right\rangle=\frac{1}{\sqrt{d_{A}}} \sum_{j=1}^{d_{A}}\left|e_{j}^{A}\right\rangle \otimes\left|e_{j}^{A}\right\rangle
$$

в $\mathscr{H}_{A} \otimes \mathscr{H}_{A}$, тогда

$$
\left(\operatorname{Id}_{A} \otimes \Phi\right)\left(\left|\Omega^{A A}\right\rangle\left\langle\Omega^{A A}\right|\right)=\frac{1}{d_{A}} \sum_{j, k=1}^{d_{A}}\left|e_{j}^{A}\right\rangle\left\langle e_{k}^{A}\right| \otimes \Phi\left(\left|e_{j}^{A}\right\rangle\left\langle e_{k}^{A}\right|\right)=\rho_{\Phi}^{A B}
$$

является положительным оператором в $\mathscr{H}_{A} \otimes \mathscr{H}_{B}$. Далее,

$$
\Phi\left(\left|e_{j}^{A}\right\rangle\left\langle e_{k}^{A}\right|\right)=d_{A}\left\langle e_{j}^{A}\left|\rho_{\Phi}^{A B}\right| e_{k}^{A}\right\rangle
$$

откуда

$$
\Phi(\rho)=d_{A} \operatorname{Tr}_{\mathscr{H}_{A}}\left(\rho^{T} \otimes I_{B}\right) \rho_{\Phi}^{A B},
$$


где $\rho^{T}$ - транспонирование оператора $\rho$ в базисе $\left\{e_{j}^{A}\right\}$. Из спектрального разложения, $d_{A} \rho_{\Phi}^{A B}=\sum_{j=1}^{N}\left|\Psi_{j}\right\rangle\left\langle\Psi_{j}\right|$, где $\Psi_{j} \neq 0$, и $N \leqslant d_{A} d_{B}$. Определяя операторы $V_{j}: \mathscr{H}_{A} \rightarrow \mathscr{H}_{B}$, действующие как

$$
V_{j}\left|\psi^{A}\right\rangle=\left\langle\bar{\psi}^{A} \mid \Psi_{j}\right\rangle
$$

где $\bar{\psi}^{A}$ - комплексное сопряжение вектора $\psi^{A} \in \mathscr{H}_{A}$ в базисе $\left\{e_{j}^{A}\right\}$, получаем (44). В случае сохранения следа получаем (45) как условие нормировки, потому что

$$
\sum_{j=1}^{N}\left\langle\psi^{A}\left|V_{j}^{*} V_{j}\right| \psi^{A}\right\rangle=\operatorname{Tr} \Phi\left(\left|\psi^{A}\right\rangle\left\langle\psi^{A}\right|\right)=\left\langle\psi^{A} \mid \psi^{A}\right\rangle .
$$

Предложение 5 доказано.

Введем унитарное пространство $\mathscr{H}_{C}$, которое является координатным $N$ мерным унитарным пространством. Для однородности обозначений положим $d_{C}=N$. Определим линейный оператор $V: \mathscr{H}_{A} \rightarrow \mathscr{H}_{B} \otimes \mathscr{H}_{C}$

$$
V=\sum_{j=1}^{d_{C}} V_{j} \otimes\left|e_{j}^{C}\right\rangle
$$

где $\left\{e_{j}^{C}\right\}$ - ортонормированный базис в $\mathscr{H}_{C}$, тогда представление Kрауса сводится $\mathrm{K}$

$$
\Phi(\rho)=\operatorname{Tr}_{\mathscr{H}_{C}} V \rho V^{*}
$$

Вообще, пусть заданы три унитарных пространства $\mathscr{H}_{A}, \mathscr{H}_{B}, \mathscr{H}_{C}$ и линейный оператор $V: \mathscr{H}_{A} \rightarrow \mathscr{H}_{B} \otimes \mathscr{H}_{C}$, тогда соотношения

$$
\Phi(\rho)=\operatorname{Tr}_{\mathscr{H}_{C}} V \rho V^{*}, \quad \widetilde{\Phi}(\rho)=\operatorname{Tr}_{\mathscr{H}_{B}} V \rho V^{*} ; \quad \rho \in \mathfrak{M}\left(\mathscr{H}_{A}\right),
$$

определяют два в.п. отображения $\Phi: \mathfrak{M}\left(\mathscr{H}_{A}\right) \rightarrow \mathfrak{M}\left(\mathscr{H}_{B}\right), \widetilde{\Phi}: \mathfrak{M}\left(\mathscr{H}_{A}\right) \rightarrow \mathfrak{M}\left(\mathscr{H}_{C}\right)$, которые мы называем комплементарными (см. [30], [31]). Если $V$ - изометрический оператор, то оба отображения сохраняют след, т.е. являются каналами. Из предложения 5 следует, что для данного в.п. отображения комплементарное всегда существует (и, в определенном смысле, единственно, см. [9], [30]).

Для любого линейного отображения $\Phi: \mathfrak{M}(\mathscr{H}) \rightarrow \mathfrak{M}\left(\mathscr{H}^{\prime}\right)$ сопряженное отображение $\Phi^{*}: \mathfrak{M}\left(\mathscr{H}^{\prime}\right) \rightarrow \mathfrak{M}(\mathscr{H})$ определяется соотношением

$$
\operatorname{Tr} \Phi(\rho) X=\operatorname{Tr} \rho \Phi^{*}(X) ; \quad \rho \in \mathfrak{M}(\mathscr{H}), \quad X \in \mathfrak{M}\left(\mathscr{H}^{\prime}\right) .
$$

Если $\Phi$ - в.п. отображение, то $\Phi^{*}$ - также в.п. отображение. Соотношения (47) равносильны соотношениям

$$
\begin{array}{ll}
\Phi^{*}(X)=V^{*}\left(X \otimes I_{C}\right) V ; & X \in \mathfrak{M}\left(\mathscr{H}_{B}\right), \\
\widetilde{\Phi}^{*}(X)=V^{*}\left(I_{B} \otimes X\right) V ; & X \in \mathfrak{M}\left(\mathscr{H}_{C}\right),
\end{array}
$$

которые описывают продолжение Стайнспринга [16] в рассматриваемом нами конечномерном случае. 
Рассматривая $\widetilde{\Phi}$ как сопряженное к в.п. отображению $\widetilde{\Phi}^{*}$, мы заключаем, что должно быть и представление вида

$$
\widetilde{\Phi}(\rho)=S_{C}\left(\rho \otimes I_{B}\right) S_{C}^{*},
$$

где $S_{C}: \mathscr{H}_{A} \otimes \mathscr{H}_{B} \rightarrow \mathscr{H}_{C}$. В случае канала условие сохранения следа сводится к $\operatorname{Tr}_{\mathscr{H}_{B}} S_{C}^{*} S_{C}=I_{A}$. Меняя ролями $\mathscr{H}_{B}, \mathscr{H}_{C}$, мы, конечно, получаем представление для исходного отображения $\Phi$, аналогичное (48), которое есть не что иное, как продолжение Стайнспринга для отображения $\Phi$, если оно рассматривается как сопряженное к $\Phi^{*}$.

Подставляя (46) во вторую формулу в (47), мы получаем явное выражение для комплементарного отображения

$$
\widetilde{\Phi}(\rho)=\sum_{j, k=1}^{d_{C}}\left|e_{j}^{C}\right\rangle\left\langle e_{k}^{C}\right| \operatorname{Tr} V_{j} \rho V_{k}^{*} ; \quad \rho \in \mathfrak{M}\left(\mathscr{H}_{A}\right)
$$

Отсюда вытекает, что представление Крауса для $\widetilde{\Phi}$ имеет вид

$$
\widetilde{\Phi}(\rho)=\sum_{k=1}^{d_{B}} \widetilde{V}_{k} \rho \widetilde{V}_{k}^{*}
$$

где операторы $\widetilde{V}_{k}: \mathscr{H}_{A} \rightarrow \mathscr{H}_{C}$ даются формулами

$$
\widetilde{V}_{k}=\sum_{j=1}^{d_{C}}\left\langle e_{k}^{B}\left|V_{j} \otimes\right| e_{j}^{C}\right\rangle
$$

и, следовательно, удовлетворяют соотношениям $\left\langle e_{j}^{C}\right| \widetilde{V}_{k}=\left\langle e_{k}^{B}\right| V_{j}$. Наконец, имеет место представление (48) для $\widetilde{\Phi}$, где

$$
S_{C}=\sum_{k=1}^{d_{B}} \widetilde{V}_{k} \otimes\left\langle e_{k}^{B}\right| .
$$

Tеорема 2 [30] (см. также [31]). Если какое-либо из соотношений

$$
\begin{gathered}
\nu_{p}\left(\Phi_{1} \otimes \Phi_{2}\right)=\nu_{p}\left(\Phi_{1}\right) \nu_{p}\left(\Phi_{2}\right), \\
\check{H}\left(\Phi_{1} \otimes \Phi_{2}\right)=\check{H}\left(\Phi_{1}\right)+\check{H}\left(\Phi_{2}\right), \\
\hat{H}_{\Phi_{1} \otimes \Phi_{2}}\left(\rho_{12}\right) \geqslant \hat{H}_{\Phi_{1}}\left(\rho_{1}\right)+\hat{H}_{\Phi_{2}}\left(\rho_{2}\right)
\end{gathered}
$$

выполняется для данных в.п. отображений (каналов) $\Phi_{1}, \Phi_{2}$, то такое же соотношение выполняется для пары комплементарных отображений $\widetilde{\Phi}_{1}, \widetilde{\Phi}_{2}$. Если какое-либо из этих соотношений выполняется для данного $\Phi_{1}$ и произвольного $\Phi_{2}$, то аналогичное соотношение выполняется для комплементарного $\widetilde{\Phi}_{1}$ и произвольного $\Phi_{2}$.

ДокАЗАТЕЛЬСтво. В силу вогнутости энтропии, величины $\nu_{p}, \check{H}$ и $\hat{H}$ не изменяются, если мы заменим в их определениях $\mathfrak{S}(\mathscr{H})$ на $\mathfrak{P}(\mathscr{H})$. Пусть 
$\rho=|\psi\rangle\langle\psi|$ для некоторого единичного вектора $|\psi\rangle \in \mathscr{H}_{A}$, тогда эрмитовы операторы $\Phi(\rho), \widetilde{\Phi}(\rho)$ имеют одинаковые ненулевые собственные значения. В самом деле, $\Phi(\rho), \widetilde{\Phi}(\rho)$ являются частичными следами оператора $\left|\psi_{B C}\right\rangle\left\langle\psi_{B C}\right|$, где $\left|\psi_{B C}\right\rangle=V|\psi\rangle \in \mathscr{H}_{B} \otimes \mathscr{H}_{C}$, и это утверждение следует из предложения 3.

$\operatorname{KaK} \operatorname{Tr} \sigma^{p}$, так и $H(\sigma)$ являются универсальными функциями ненулевых собственных значений эрмитова оператора $\sigma$. Поэтому из определений величин $\nu_{p}$, $\check{H}$ и $\hat{H}$ вытекает, что для произвольного в.п. отображения $\Phi$

$$
\nu_{p}(\widetilde{\Phi})=\nu_{p}(\Phi)
$$

Более того, если $\Phi-$ канал, то

$$
\begin{aligned}
\check{H}(\widetilde{\Phi}) & =\check{H}(\Phi), \\
\hat{H}_{\widetilde{\Phi}}(\rho) & =\hat{H}_{\Phi}(\rho) .
\end{aligned}
$$

Теперь заметим, что если $\Phi_{j}, \widetilde{\Phi}_{j}, j=1,2$, - две пары комплементарных отображений, то отображения $\Phi_{1} \otimes \Phi_{2}$ и $\widetilde{\Phi}_{1} \otimes \widetilde{\Phi}_{2}$ также комплементарны. Для доказательства достаточно положить $\mathscr{H}_{B}=\mathscr{H}_{B_{1}} \otimes \mathscr{H}_{B_{2}}, \mathscr{H}_{C}=\mathscr{H}_{C_{1}} \otimes \mathscr{H}_{C_{2}}$ и $V=V_{1} \otimes V_{2}$. Суммируя все сказанное, получаем утверждение теоремы. Теорема 2 доказана.

\section{5. Мультипликативность/аддитивность для некоторых классов каналов}

Выполнимость гипотезы мультипликативности (21) для всех $p \geqslant 1$ и гипотез аддитивности (25), (27) была установлена для ряда случаев, когда один из каналов произволен, а другой принадлежит одному из классов, которые мы рассмотрим ниже.

\section{1. Тождественный канал и связанные с ним каналы.}

ЛЕмма 2. Для любого в.п. отображения $\Phi$ u $p \geqslant 1$

$$
\nu_{p}(\Phi \otimes \mathrm{Id})=\nu_{p}(\Phi) .
$$

Поскольку $\nu_{p}(\mathrm{Id})=1$, это соотношение действительно означает мультипликативность (21).

ДокАЗАТЕЛЬство. Обозначим $\mathscr{H}_{1}, \mathscr{H}_{2}$ (входные) пространства отображений $\Phi$ и Id соответственно. Пусть $\psi_{12}-$ единичный вектор в $\mathscr{H}_{1} \otimes \mathscr{H}_{2}$, тогда $\rho_{12}=\left|\psi_{12}\right\rangle\left\langle\psi_{12}\right|$ и $\rho_{1}=\operatorname{Tr}_{2} \rho_{12}$ - частичное состояние в $\mathscr{H}_{1}$. Обозначим $\rho_{12}^{\prime}=(\Phi \otimes \mathrm{Id})\left(\rho_{12}\right)$. Используем представление $(47)$ для отображения $\Phi$. Рассмотрим оператор $V: \mathscr{H}_{1} \rightarrow \mathscr{H}_{1}^{\prime} \otimes \mathscr{H}_{3}$ из этого представления, где $\mathscr{H}_{1}^{\prime}$ - выходное пространство $\Phi$ и $\mathscr{H}_{3}=\mathscr{H}_{C}$. Вектор $|\psi\rangle=\left(V \otimes I_{2}\right)\left|\psi_{12}\right\rangle$ определяет оператор $\rho_{123}^{\prime}=|\psi\rangle\langle\psi|$ в пространстве $\mathscr{H}_{1}^{\prime} \otimes \mathscr{H}_{2} \otimes \mathscr{H}_{3}$. Согласно предложению 3 , частичные следы $\rho_{12}^{\prime}, \rho_{3}^{\prime}$ имеют одинаковый ненулевой спектр. Поэтому

$$
\left\|(\Phi \otimes \operatorname{Id})\left(\rho_{12}\right)\right\|_{p}=\left\|\rho_{12}^{\prime}\right\|_{p}=\left\|\rho_{3}^{\prime}\right\|_{p}=\left\|\Psi\left(\rho_{1}\right)\right\|_{p},
$$

где в.п. отображение $\Psi: \mathfrak{M}\left(\mathscr{H}_{1}\right) \rightarrow \mathfrak{M}\left(\mathscr{H}_{3}\right)$ определяется как

$$
\Psi\left(\rho_{1}\right)=\operatorname{Tr}_{\mathscr{H}_{1}^{\prime} \otimes \mathscr{H}_{2}}\left(V \otimes I_{2}\right)\left|\psi_{12}\right\rangle\left\langle\psi_{12}\right|\left(V \otimes I_{2}\right)=\rho_{3}^{\prime} .
$$


Теперь заметим, что отображение $\rho_{1} \rightarrow \Psi\left(\rho_{1}\right)$ аффинно, а норма - выпукла, поэтому максимум этой величины достигается на чистом состоянии $\rho_{1}$, откуда $\rho_{12}=\rho_{1} \otimes \rho_{2}$, и утверждение доказано. Лемма 2 доказана.

Пусть $\Phi-$ канал. Переходя к пределу $p \downarrow 1$, мы получаем

$$
\check{H}(\Phi \otimes \mathrm{Id})=\check{H}(\Phi)
$$

что означает аддитивность $(25)$, поскольку очевидно, что $\check{H}(\mathrm{Id})=0$.

Эти результаты могут быть легко распространены на более широкий класс каналов.

СЛЕДСТвИЕ 1 [32]. Гипотезы мультипликативности (21) и аддитивности (25) выполняются для произвольного канала $\Phi_{1}$ и канала $\Phi_{2}$ такого, что

$$
\nu_{p}\left(\Phi_{2}\right)=1, \quad \text { соответственно } \check{H}\left(\Phi_{2}\right)=0 .
$$

ДокАЗАТЕЛЬСтво. Имеем

$$
\nu_{p}\left(\Phi_{1} \otimes \Phi_{2}\right)=\nu_{p}\left(\left(\Phi_{1} \otimes \operatorname{Id}_{2}\right) \circ\left(\operatorname{Id}_{1} \otimes \Phi_{2}\right)\right) \leqslant \nu_{p}\left(\Phi_{1} \otimes \operatorname{Id}_{2}\right),
$$

поскольку образ пространства состояний при отображении $\operatorname{Id}_{1} \otimes \Phi_{2}$ содержится в пространстве состояний. Применяя (54), получаем

$$
\nu_{p}\left(\Phi_{1} \otimes \operatorname{Id}_{2}\right)=\nu_{p}\left(\Phi_{1}\right)=\nu_{p}\left(\Phi_{1}\right) \nu_{p}\left(\Phi_{2}\right) .
$$

Поэтому $\nu_{p}\left(\Phi_{1} \otimes \Phi_{2}\right) \leqslant \nu_{p}\left(\Phi_{1}\right) \nu_{p}\left(\Phi_{2}\right)$, откуда следует мультипликативность. Аддитивность доказывается аналогичным образом. Следствие 1 доказано.

Комплементарным к идеальному каналу является полностъю деполяризующий канал

$$
\Phi(\rho)=\frac{I}{d} \operatorname{Tr} \rho,
$$

простейший из целого класса каналов, который мы рассмотрим далее.

5.2. Каналы, разрушающие сцепленность, и комплементарные к ним. Любое линейное отображение $\Phi: \mathfrak{M}(\mathscr{H}) \rightarrow \mathfrak{M}\left(\mathscr{H}^{\prime}\right)$ допускает представление

$$
\Phi(X)=\sum_{j} N_{j}^{\prime} \operatorname{Tr} X M_{j},
$$

где $\left\{M_{j}\right\},\left\{N_{j}^{\prime}\right\}$ - конечные наборы операторов в $\mathscr{H}$ и в $\mathscr{H}^{\prime}$ соответственно. Это просто следует из конечномерности пространств $\mathscr{H}, \mathscr{H}^{\prime}$ и того факта, что любой линейный функционал на $\mathfrak{M}(\mathscr{H})$ имеет вид $X \rightarrow \operatorname{Tr} X M$, где $M \in$ $\mathfrak{M}(\mathscr{H})$.

ПрЕДЛОЖЕНИЕ 6. Для данного линейного отображения $\Phi: \mathfrak{M}(\mathscr{H}) \rightarrow$ $\mathfrak{M}\left(\mathscr{H}^{\prime}\right)$ следующие условия равносильны:

(i) найдется представление (55) такое, что $M_{j} \geqslant 0, N_{j}^{\prime} \geqslant 0$;

(ii) отображение $\Phi$ вполне положительно и имеет представление Крауса (44) с операторами $V_{\alpha}$ ранга 1 ,

$$
\Phi(\rho)=\sum_{\alpha=1}^{\widetilde{d}}\left|\varphi_{\alpha}\right\rangle\left\langle\psi_{\alpha}|\rho| \psi_{\alpha}\right\rangle\left\langle\varphi_{\alpha}\right|,
$$

где $\left\{\psi_{\alpha}\right\} \in \mathscr{H},\left\{\varphi_{\alpha}\right\} \in \mathscr{H}^{\prime}-$ некоторые наборы векторов; 
(iii) для $d=2,3, \ldots$ июбого $\rho_{12} \in \mathfrak{S}\left(\mathscr{H} \otimes \mathscr{H}_{d}\right)$

$$
\left(\Phi \otimes \operatorname{Id}_{d}\right)(\rho)=\sum_{\alpha} A_{\alpha} \otimes B_{\alpha}
$$

где $A_{\alpha} \geqslant 0$ u $B_{\alpha} \geqslant 0$.

Каналы, удовлетворяющие условию (i), были введены в работе [26], а сформулированная выше характеризация получена в [33]. Следуя [33], мы будем называть такие отображения разрушающими сцепленность (р.c.). Отображение (56) является каналом тогда и только тогда, когда выполняется соотношение полноты

$$
\sum_{\alpha=1}^{\widetilde{d}}\left|\psi_{\alpha}\right\rangle\left\langle\varphi_{\alpha} \mid \varphi_{\alpha}\right\rangle\left\langle\psi_{\alpha}\right|=I
$$

В этом случае (57) означает, что выходное состояние $\left(\Phi \otimes \operatorname{Id}_{d}\right)(\rho)$ всегда разделимо, т.е. является выпуклой комбинацией (несцепленных) состояний-произведений.

ПримечАние. Р.с. канал может быть записан в виде

$$
\Phi(\rho)=\sum_{j} \rho_{j}^{\prime} \operatorname{Tr} \rho M_{j}
$$

где $\left\{\rho_{j}^{\prime}\right\}$ - конечный набор состояний в $\mathscr{H}^{\prime}$, а $\left\{M_{j}\right\}$ - разложение единицы в $\mathscr{H}$, т.е. набор операторов, удовлетворяющих условиям

$$
M_{j} \geqslant 0, \quad \sum_{j} M_{j}=I .
$$

Разложения единицы описывают квантовые наблюдаемые [1], а канал (58) описывает измерение наблюдаемой $\left\{M_{j}\right\}$ в системе с входным состоянием $\rho$, с результирующим распределением вероятностей $\left\{\operatorname{Tr} \rho M_{j}\right\}$ и последующим приготовлением одного из выходных состояний $\rho_{j}^{\prime}$. Таким образом, действие канала предполагает классическую обработку информации, что и приводит к разрушению сцепленности.

ДокАЗАТЕЛЬСтво. $\mathrm{i} \Rightarrow \mathrm{ii}$ следует из спектрального разложения операторов $M_{j}, N_{j}^{\prime}$.

$\mathrm{ii} \Rightarrow$ iii получается непосредственно, если положить $A_{\alpha}=\left|\psi_{\alpha}\right\rangle\left\langle\psi_{\alpha}\right|, B_{\alpha}=$ $\left\langle\varphi_{\alpha}\left|\rho_{12}\right| \varphi_{\alpha}\right\rangle$.

$\mathrm{iii} \Rightarrow \mathrm{i}$ Рассмотрим максимально сцепленный вектор

$$
\left|\Omega^{A A}\right\rangle=\frac{1}{\sqrt{d_{A}}} \sum_{j=1}^{d_{A}}\left|e_{j}^{A}\right\rangle \otimes\left|e_{j}^{A}\right\rangle
$$

в $\mathscr{H}_{A} \otimes \mathscr{H}_{A}$, тогда

$$
\left(\Phi \otimes \operatorname{Id}_{A}\right)\left(\left|\Omega^{A A}\right\rangle\left\langle\Omega^{A A}\right|\right)=\frac{1}{d_{A}} \sum_{j, k=1}^{d_{A}} \Phi\left(\left|e_{j}^{A}\right\rangle\left\langle e_{k}^{A}\right|\right) \otimes\left|e_{j}^{A}\right\rangle\left\langle e_{k}^{A}\right|=\sum_{\alpha} A_{\alpha} \otimes B_{\alpha}
$$


откуда $\Phi\left(\left|e_{j}^{A}\right\rangle\left\langle e_{k}^{A}\right|\right)=d_{A} \sum_{\alpha} A_{\alpha}\left\langle e_{j}^{A}\left|B_{\alpha}\right| e_{k}^{A}\right\rangle=\Phi^{\prime}\left(\left|e_{j}^{A}\right\rangle\left\langle e_{k}^{A}\right|\right)$, где $\Phi^{\prime}$ - p.с. отображение, определяемое соотношением

$$
\Phi^{\prime}(\rho)=d_{A} \sum_{\alpha} A_{\alpha} \operatorname{Tr} \rho B_{\alpha}^{T},
$$

причем ${ }^{T}$ обозначает транспонирование в базисе $\left\{e_{j}^{A}\right\}$. Предложение 6 доказано.

Согласно соотношению (49), комплементарное к р.с. отображению (56) имеет вид

$$
\widetilde{\Phi}(\rho)=\sum_{j, k=1}^{d_{C}} c_{j k}\left\langle\psi_{j}|\rho| \psi_{k}\right\rangle\left|e_{j}^{C}\right\rangle\left\langle e_{k}^{C}\right| ; \quad \rho \in \mathfrak{M}\left(\mathscr{H}_{A}\right),
$$

где $c_{j k}=\left\langle\varphi_{k} \mid \varphi_{j}\right\rangle$. Заметим, что, согласно известной лемме Колмогорова, произвольная неотрицательно определенная матрица $\left[c_{j k}\right]$ может быть представлена в таком виде. В частном случае, когда $\left\{\psi_{j}\right\}_{j=\overline{1, d_{C}}}$ ортонормированный базис в $\mathscr{H}$, соотношение (59) задает диагональное в.п. отображение [34]. Диагональные каналы характеризуются дополнительным свойством $c_{j j} \equiv 1$.

Представляя $c_{j k}=\sum_{l=1}^{d_{C}} \bar{\varphi}_{k l} \varphi_{j l}$ и обозначая

$$
\widetilde{V}_{k}=\sum_{j=1}^{d_{C}} \varphi_{j k}\left|e_{j}\right\rangle\left\langle\psi_{j}\right|,
$$

получаем представление Крауса (50) для комплементарного отображения. Для диагональных отображений $\left|\psi_{j}\right\rangle=\left|e_{j}\right\rangle$, поэтому из (60) видно, что диагональные отображения характеризуются тем, что имеют представление Крауса с одновременно диагонализуемыми (т.е. коммутирующими нормальными) операторами $\widetilde{V}_{k}$.

Для р.с. каналов свойство аддитивности (25) (а также и (30), хотя и в неявной форме) с произвольным вторым каналом было доказано в работе П. Шоpa [35], а свойство мультипликативности (21) для всех $p>1$ - в работе К. Кинга [36] с использованием неравенства Либа-Тирринга. Это доказательство почти без изменений переносится на случай р.с. отображений.

Неравенство Либа-Тирринга состоит в следующем.

Tеорема 3 [37]. Для любых операторов $A, B \in \mathfrak{M}(\mathscr{H})$ таких, что $A, B \geqslant 0$, u $p \geqslant 1$ выполняется

$$
\operatorname{Tr}(A B)^{p} \leqslant \operatorname{Tr} A^{p} B^{p} .
$$

ДоказАтеЛЬство. Сначала докажем, что

$$
\|A B\|^{p} \leqslant\left\|A^{p} B^{p}\right\|, \quad p \geqslant 1 .
$$

Положим $C=A^{p} / \sqrt{\left\|A^{p} B^{p}\right\|}, D=B^{p} / \sqrt{\left\|A^{p} B^{p}\right\|}$, тогда для этого достаточно доказать, что $C, D \geqslant 0$ и $\|C D\| \leqslant 1$ влечет

$$
\left\|C^{s} D^{s}\right\| \leqslant 1, \quad s \in[0,1] .
$$

В силу свойств операторной нормы,

$$
\|C D\| \leqslant 1 \Leftrightarrow\left\|D C^{2} D\right\| \leqslant 1 \Leftrightarrow D C^{2} D \leqslant I .
$$


Не ограничивая общности, мы можем предположить, что $D$ невырожден. Тогда $D C^{2} D \leqslant I \Rightarrow C^{2} \leqslant D^{-2}$, и в силу операторной монотонности функции $x \rightarrow x^{s}$, $s \in[0,1]$ [38; теорема V.1.9], имеет место $C^{2 s} \leqslant D^{-2 s}$. Рассуждая как в (64), но в обратном направлении, мы получаем (63), а следовательно, и (62).

Дальнейшее доказательство следует работе Х. Араки [39], см. также [38; теорема IX.2.10]. Для положительного оператора $X \in \mathfrak{M}(\mathscr{H})$ и $k=1, \ldots, d$ рассмотрим $k$-ю антисимметричную (грассманову) тензорную степень $X^{\wedge k}$ (которая равна $X^{\otimes k} P_{-}$, где $P_{-}-$проектор на подпространство $\mathscr{H}^{\wedge k}$ антисимметричных векторов из $\mathscr{H}^{\otimes k}$ ). Нам потребуются следующие свойства (см. [38; раздел I.5]):

$$
\left\|X^{\wedge k}\right\|=\lambda_{1} \cdots \lambda_{k},
$$

где $\lambda_{1} \geqslant \cdots \geqslant \lambda_{d}$ - собственные значения оператора $X$ (с учетом кратности) и $\left(X^{\wedge k}\right)^{p}=\left(X^{p}\right)^{\wedge k}$. Рассмотрим операторы

$$
Y_{p}=A^{p / 2} B^{p / 2}, \quad X_{p}=Y_{p}^{*} Y_{p}=B^{p / 2} A^{p} B^{p / 2}
$$

и обозначим собственные значения операторов $X_{p}$ и $X_{1}$ соответственно через

$$
\lambda_{1} \geqslant \cdots \geqslant \lambda_{d}, \quad \mu_{1} \geqslant \cdots \geqslant \mu_{d}
$$

Тогда

$$
\left\|X_{p}^{\wedge k}\right\|=\lambda_{1} \cdots \lambda_{k}, \quad\left\|X_{1}^{\wedge k}\right\|=\mu_{1} \cdots \mu_{k} .
$$

С другой стороны,

$$
\left\|X_{p}^{\wedge k}\right\|=\left\|\left(Y_{p}^{\wedge k}\right)^{*} Y_{p}^{\wedge k}\right\|=\left\|Y_{p}^{\wedge k}\right\|^{2}=\left\|\left[\left(A^{1 / 2}\right)^{\wedge k}\right]^{p}\left[\left(B^{1 / 2}\right)^{\wedge k}\right]^{p}\right\|^{2}
$$

и поэтому

$$
\left\|X_{1}^{\wedge k}\right\|=\left\|\left(A^{1 / 2}\right)^{\wedge k}\left(B^{1 / 2}\right)^{\wedge k}\right\|^{2} .
$$

В силу (62),

$$
\left\|X_{1}^{\wedge k}\right\|^{p} \leqslant\left\|X_{p}^{\wedge k}\right\|
$$

т.е.

$$
\mu_{1}^{p} \cdots \mu_{k}^{p} \leqslant \lambda_{1} \cdots \lambda_{k} ; \quad k=1, \ldots, d
$$

Но это отношение мажоризации влечет (см., например, [38; пример II.3.5])

$$
\sum_{j=1}^{k} \mu_{j}^{p} \leqslant \sum_{j=1}^{k} \lambda_{j} ; \quad k=1, \ldots, d
$$

в частности, для $k=d$ выполнено $\operatorname{Tr} X_{1}^{p} \leqslant \operatorname{Tr} X_{p}$ или

$$
\operatorname{Tr}\left(B^{1 / 2} A B^{1 / 2}\right)^{p} \leqslant \operatorname{Tr} B^{p / 2} A^{p} B^{p / 2},
$$

что равносильно соотношению (61). На самом деле, отношение мажоризации (65) влечет гораздо более общее операторное неравенство (см. [39], [38]), но оно нам не понадобится. Теорема 3 доказана.

ПреДЛОЖЕНИЕ 7 [36]. Гипотеза мулътипликативности (21) с $p \geqslant 1$ въполняется для любого р.с. отображения $\Phi_{1}$ и произвольного в.п. отображения $\Phi_{2}$. 
ДокАЗАТЕЛЬство. Пусть $\rho_{12} \in \mathscr{H}_{1} \otimes \mathscr{H}_{2}-$ положительный оператор, тогда $\left(\Phi_{1} \otimes \mathrm{Id}_{2}\right)\left(\rho_{12}\right)=\sum_{j} A_{j} \otimes B_{j}$, где $A_{j} \geqslant 0$ и $B_{j} \geqslant 0$, и мы можем предположить, что $\operatorname{Tr} B_{j}=1$ для всех $j$. Тогда

$$
\left(\Phi_{1} \otimes \Phi_{2}\right)\left(\rho_{12}\right)=\sum_{j} A_{j} \otimes \Phi_{2}\left(B_{j}\right) .
$$

Это выражение может быть представлено в виде $V B V^{*}$, где

$$
V=\left[\sqrt{A_{1}} \otimes I_{2} \ldots \sqrt{A_{N}} \otimes I_{2}\right], \quad B=\operatorname{diag}\left[I_{1} \otimes \Phi_{2}\left(B_{j}\right)\right] .
$$

В силу полярного разложения $V$, матрицы $V B V^{*}$ и $B\left(V^{*} V\right)$ имеют одинаковые ненулевые собственные значения. Поэтому

$$
\operatorname{Tr}\left(\Phi_{1} \otimes \Phi_{2}\right)\left(\rho_{12}\right)^{p}=\operatorname{Tr}\left(V B V^{*}\right)^{p}=\operatorname{Tr}\left(B V^{*} V\right)^{p}
$$

Применяя неравенство Либа-Тирринга, получаем

$$
\begin{aligned}
\operatorname{Tr}\left(\Phi_{1} \otimes \Phi_{2}\right)\left(\rho_{12}\right)^{p} & \leqslant \operatorname{Tr} B^{p}\left(V^{*} V\right)^{p} \\
& =\sum_{j=1}^{N} \operatorname{Tr}\left(\left(R^{*} R\right)^{p}\right)_{j j} \operatorname{Tr} \Phi_{2}\left(B_{j}\right)^{p},
\end{aligned}
$$

где $R=\left[\sqrt{A_{1}} \ldots \sqrt{A_{N}}\right]$. Поскольку $\operatorname{Tr} B_{j}=1$, имеем $\operatorname{Tr}\left(\Phi_{2}\left(B_{j}\right)\right)^{p} \leqslant \nu_{p}\left(\Phi_{2}\right)^{p}$, тогда как

$$
\sum_{j=1}^{N} \operatorname{Tr}\left(\left(R^{*} R\right)^{p}\right)_{j j}=\operatorname{Tr}\left(R^{*} R\right)^{p}=\operatorname{Tr}\left(R R^{*}\right)^{p} .
$$

Однако, беря второй частичный след в (66), получаем

$$
\Phi_{1}\left(\rho_{1}\right)=\sum_{j} A_{j}=R R^{*}
$$

откуда $\operatorname{Tr}\left(R R^{*}\right)^{p}=\operatorname{Tr} \Phi_{1}\left(\rho_{1}\right)^{p} \leqslant \nu_{p}\left(\Phi_{1}\right)^{p}$. Поэтому

$$
\left\|\left(\Phi_{1} \otimes \Phi_{2}\right)\left(\rho_{12}\right)\right\|^{p} \leqslant \nu_{p}\left(\Phi_{1}\right) \nu_{p}\left(\Phi_{2}\right)
$$

для произвольного $\rho_{12}$. Предложение 7 доказано.

Таким образом, мы установили свойство мультипликативности (13) для р.с. отображений (каналов) и, следовательно, по теореме 2, для комплементарных им отображений, которые имеют вид (59). Отсюда следует и аддитивность минимальной выходной энтропии (25) для таких каналов.

ПрЕДЛОЖЕНИЕ 8. Неравенство (30) выполняется, если $\Phi_{1}$ - р.с. канал или комплементарный к нему, а $\Phi_{2}$ - произвольный канал.

Отсюда следует, что в этом случае имеет место и аддитивность $\chi$-пропускной способности (27).

Нам понадобится следующая лемма. 
ЛЕмма 3. Пусть $\pi_{j} \geqslant 0, \sum \pi_{j}=1 u \rho_{1}^{j}, \rho_{2}^{j}-$ произвольные состояния квантовых систем 1, 2. Тогда

$$
H\left(\sum_{j} \pi_{j} \rho_{1}^{j} \otimes \rho_{2}^{j}\right) \geqslant H\left(\sum_{j} \pi_{j} \rho_{1}^{j}\right)+\sum_{j} \pi_{j} H\left(\rho_{2}^{j}\right) .
$$

ДокАЗАТЕЛЬство. Введем систему 3, ортонормированный базис которой нумеруется индексом $j$, и рассмотрим состояние составной системы 123 вида

$$
\rho_{123}=\sum_{j} \pi_{j} \rho_{1}^{j} \otimes \rho_{2}^{j} \otimes\left|e_{j}\right\rangle\left\langle e_{j}\right| .
$$

Мы будем использовать фундаментальное свойство сильной субаддитивности квантовой энтропии, доказанное Э. Либом и М. Б. Рускаи (см., например, [13], [1], [3]): для произвольного состояния $\rho_{123}$

$$
H\left(\rho_{12}\right)+H\left(\rho_{13}\right) \geqslant H\left(\rho_{123}\right)+H\left(\rho_{1}\right) .
$$

Вычисляя частичные следы, получаем выражения

$$
\begin{aligned}
& H\left(\rho_{12}\right)=H\left(\sum_{j} \pi_{j} \rho_{1}^{j} \otimes \rho_{2}^{j}\right), \quad H\left(\rho_{1}\right)=H\left(\sum_{j} \pi_{j} \rho_{1}^{j}\right), \\
& H\left(\rho_{123}\right)=\sum_{j} \pi_{j}\left[H\left(\rho_{1}^{j}\right)+H\left(\rho_{2}^{j}\right)\right]-\sum_{j} \pi_{j} \log \pi_{j}, \\
& H\left(\rho_{13}\right)=\sum_{j} \pi_{j} H\left(\rho_{1}^{j}\right)-\sum_{j} \pi_{j} \log \pi_{j},
\end{aligned}
$$

подставляя которые в (67), получаем утверждение леммы. Лемма 3 доказана.

Возвращаясь к доказательству предложения 8 , предположим, что $\Phi_{1}-$ p.c. канал в системе 1 , так что $\Phi_{1}(\rho)=\sum_{j} \rho_{1}^{j} \operatorname{Tr} \rho M_{1}^{j}$, где $\left\{M_{1}^{j}\right\}$ является наблюдаемой. Тогда для произвольного состояния $\rho_{12}$ составной системы

$$
\left(\Phi_{1} \otimes \operatorname{Id}_{2}\right)\left(\rho_{12}\right)=\sum_{j} p_{j} \rho_{1}^{j} \otimes \rho_{2}^{j},
$$

где вероятности $p_{j}$ и операторы плотности $\rho_{2}^{j}$ определяются соотношениями $p_{j} \rho_{2}^{j}=\operatorname{Tr}_{\mathscr{H}_{1}} \rho_{12}\left(M_{1}^{j} \otimes I_{2}\right)$. Беря частичный след, получаем, в частности,

$$
\Phi_{1}\left(\operatorname{Tr}_{\mathscr{H}_{2}} \rho_{12}\right)=\sum_{j} p_{j} \rho_{1}^{j} .
$$

Если $\Phi_{2}-$ произвольный канал в системе 2, то

$$
\left(\Phi_{1} \otimes \Phi_{2}\right)\left(\rho_{12}\right)=\sum_{j} p_{j} \rho_{1}^{j} \otimes \Phi_{2}\left(\rho_{2}^{j}\right) .
$$

Пусть теперь $\left\{\pi_{i}\right\}$ - распределение вероятностей и $\left\{\rho_{12}^{i}\right\}$ - семейство состояний такое, что $\rho_{12}=\sum_{i} \pi_{i} \rho_{12}^{i}$ и

$$
\hat{H}_{\Phi_{1} \otimes \Phi_{2}}\left(\rho_{12}\right)=\sum_{i} \pi_{i} H\left(\left(\Phi_{1} \otimes \Phi_{2}\right)\left(\rho_{12}^{i}\right)\right)=\sum_{i} \pi_{i} H\left(\sum_{j} p_{i j} \rho_{1}^{j} \otimes \Phi_{2}\left(\rho_{2}^{i j}\right)\right),
$$


где $p_{i j} \rho_{2}^{i j}=\operatorname{Tr}_{\mathscr{H}_{1}} \rho_{12}^{i}\left(M_{1}^{j} \otimes I_{2}\right)$. Используя лемму, мы видим, что это больше или равно, чем

$$
\sum_{i} \pi_{i} H\left(\sum_{j} p_{i j} \rho_{1}^{j}\right)+\sum_{i j} \pi_{i} p_{i j} H\left(\Phi_{2}\left(\rho_{2}^{i j}\right)\right),
$$

где $\sum_{j} p_{i j} \rho_{1}^{j}=\Phi_{1}\left(\operatorname{Tr}_{\mathscr{H}_{2}} \rho_{12}^{i}\right)$ согласно (68), что больше или равно, чем $\hat{H}_{\Phi_{1}}\left(\rho_{1}\right)+$ $\hat{H}_{\Phi_{2}}\left(\rho_{2}\right)$.

Утверждение для комплементарных каналов следует из теоремы 2. Предложение 8 доказано.

5.3. Ортогональные выпуклые суммы каналов. Пусть $\left\{p_{j}\right\}$ - конечное распределение вероятностей и $\Phi_{j}: \mathfrak{M}(\mathscr{H}) \rightarrow \mathfrak{M}\left(\mathscr{H}_{j}^{\prime}\right)$ - семейство каналов. Канал $\Phi: \mathfrak{M}(\mathscr{H}) \rightarrow \mathfrak{M}\left(\bigoplus_{j} \mathscr{H}_{j}^{\prime}\right)$ назовем ортогональной выпуклой суммой каналов $\Phi_{j}$,

$$
\Phi=\bigoplus_{j} p_{j} \Phi_{j}
$$

если $\Phi(\rho)=\bigoplus_{j} p_{j} \Phi_{j}(\rho)$ для всех $\rho \in \mathfrak{S}(\mathscr{H})$.

ПреДЛОЖеНИЕ 9 [40]. Пусть $\Phi_{2}$ - произволъный канал. Свойства (21), (25), (30) выполняются, если $\Phi_{1}$ - ортогональная выпуклая сумма идеального либо полностью деполяризующего канала и канала $\Phi^{(0)}$ такого, что соответствующее свойство выполняется для $\Phi^{(0)} u \Phi_{2}$.

Отсюда следует аддитивность $\chi$-пропускной способности (27) для этих классов каналов.

Таким образом мы, например, получаем все свойства мультипликативности/ аддитивности для еще одного важного случая - стирающего канала

$$
\Phi(\rho)=\left[\begin{array}{cc}
p \rho & 0 \\
0 & (1-p) \operatorname{Tr} \rho
\end{array}\right]
$$

поскольку он является ортогональной выпуклой суммой идеального и полностью деполяризующего каналов.

ДокАЗАТЕЛЬство. Мы ограничимся доказательством свойства (30), поскольку остальные свойства доказываются аналогично.

Обозначим $\Phi^{(q)}=q \operatorname{Id} \oplus(1-q) \Phi^{(0)}$. Тогда

$$
H\left(\Phi^{(q)}(\rho)\right)=q H(\rho)+(1-q) H\left(\Phi^{(0)}(\rho)\right)+h_{2}(q),
$$

где $h_{2}(q)=-q \log q-(1-q) \log (1-q)-$ двоичная энтропия, и

$$
\hat{H}_{\Phi^{(q)}}(\rho)=(1-q) \hat{H}_{\Phi^{(0)}}(\rho)+h_{2}(q),
$$

потому что минимум в выражении для $\hat{H}_{\Phi(0)}(\rho)$ достигается на ансамбле чистых состояний $\rho_{j}$, для которых $H\left(\rho_{j}\right)=0$. Для произвольного канала $\Phi_{2}$ имеем

$$
\Phi^{(q)} \otimes \Phi_{2}=q\left(\operatorname{Id} \otimes \Phi_{2}\right) \oplus(1-q)\left(\Phi^{(0)} \otimes \Phi_{2}\right) .
$$


Тогда

$$
\begin{aligned}
\hat{H}_{\Phi^{(q)} \otimes \Phi_{2}}\left(\rho_{12}\right) & \geqslant q \hat{H}_{\mathrm{Id} \otimes \Phi_{2}}\left(\rho_{12}\right)+(1-q) \hat{H}_{\Phi^{(0)} \otimes \Phi_{2}}\left(\rho_{12}\right)+h_{2}(q) \\
& \geqslant q \hat{H}_{\Phi_{2}}\left(\rho_{2}\right)+(1-q)\left[\hat{H}_{\Phi^{(0)}}\left(\rho_{1}\right)+\hat{H}_{\Phi_{2}}\left(\rho_{2}\right)\right]+h_{2}(q),
\end{aligned}
$$

где мы использовали супераддитивность величин $\hat{H}_{\mathrm{Id} \otimes \Phi_{2}}\left(\rho_{12}\right), \hat{H}_{\Phi^{(0)} \otimes \Phi_{2}}\left(\rho_{12}\right)$ и тот факт, что $\hat{H}_{\text {Id }}\left(\rho_{1}\right) \equiv 0$. Используя $(70)$, получаем, что правая часть равна $\hat{H}_{\Phi^{(q)}}\left(\rho_{1}\right)+\hat{H}_{\Phi_{2}}\left(\rho_{2}\right)$. Предложение 9 доказано.

5.4. Ковариантные каналы. Рассмотрим группу $G$ (конечную либо непрерывную), и пусть $g \rightarrow U_{g}^{A}, U_{g}^{B} ; g \in G$, - два унитарных (проективных) представления $G$ в $\mathscr{H}_{A}, \mathscr{H}_{B}$ (см., например, [41]). В.п. отображение $\Phi: \mathfrak{M}\left(\mathscr{H}_{A}\right) \rightarrow$ $\mathfrak{M}\left(\mathscr{H}_{B}\right)$ называется ковариантным, если

$$
\Phi\left(U_{g}^{A} \rho U_{g}^{A *}\right)=U_{g}^{B} \Phi(\rho) U_{g}^{B *}
$$

для всех $g \in G$ и всех $\rho$. Структура ковариантных в.п. отображений изучалась в контексте ковариантных динамических полугрупп (см., например, [42]). В частности, для ковариантного в.п. отображения существует ковариантное продолжение Стайнспринга: а именно, существует проективное представление $g \rightarrow U_{g}^{C}$ в $\mathscr{H}_{B}$ такое, что

$$
\left(U_{g}^{B} \otimes U_{g}^{C}\right) V=V U_{g}^{A}
$$

Отсюда следует, что комплементарное отображение также ковариантно:

$$
\widetilde{\Phi}\left(U_{g}^{A} \rho U_{g}^{A *}\right)=U_{g}^{C} \widetilde{\Phi}(\rho) U_{g}^{C *} .
$$

Лемма 4. Если представление $U_{g}^{A}$ неприводимо, то

$$
C_{\chi}(\Phi)=H\left(\Phi\left(\frac{I_{A}}{d_{A}}\right)\right)-\check{H}(\Phi) .
$$

ДокАзАтельство. Предположим сначала, что группа конечна. Из соотношений ортогональности для неприводимых представлений (см., например, [41]) имеем

$$
|G|^{-1} \sum_{g \in G} U_{g}^{A} \rho U_{g}^{A *}=\frac{I_{A}}{d_{A}} .
$$

Прежде всего, покажем, что

$$
\max _{\rho \in \mathfrak{P}(\mathscr{H})} H(\Phi(\rho))=H\left(\Phi\left(\frac{I_{A}}{d_{A}}\right)\right) .
$$

Это следует из того, что функция $\rho \rightarrow H(\Phi(\rho))$ является вогнутой и инвариантной относительно преобразований $\rho \rightarrow U_{g}^{A} \rho U_{g}^{A *}$, поэтому для любого $\rho$

$$
H(\Phi(\rho))=|G|^{-1} \sum_{g \in G} H\left(\Phi\left(U_{g}^{A} \rho U_{g}^{A *}\right)\right) \leqslant H\left(\Phi\left(|G|^{-1} \sum_{g \in G} U_{g}^{A} \rho U_{g}^{A *}\right)\right) .
$$


Неравенство $\leqslant$ следует тогда из (73), (28), и достаточно показать, что

$$
C_{\chi}(\Phi) \geqslant H\left(\Phi\left(\frac{I_{A}}{d_{A}}\right)\right)-\check{H}(\Phi) .
$$

Выберем состояние $\rho_{0}$, минимизирующее выходную энтропию. Тогда значение в правой части (74) достигается в формуле (26) для ансамбля состояний $\rho_{g}=$ $U_{g}^{A} \rho_{0} U_{g}^{A *} ; g \in G$, с равными вероятностями $\pi_{g}=|G|^{-1}$.

Если группа непрерывна, применимы аналогичные рассуждения, но с непрерывным оптимизирующим распределением, а именно с равномерным распределением на $G$. Затем следует аппроксимировать его конечными распределениями и воспользоваться свойствами непрерывности. Лемма 4 доказана.

Поскольку тензорное произведение неприводимых представлений (возможно, различных) групп симметрий $G_{1}, G_{2}$ является неприводимым представлением группы $G_{1} \times G_{2}$, получаем, что свойства аддитивности (25) и (27) равносильны для пары каналов, удовлетворяющих условию леммы 4. Соображения симметрии позволяют вычислить в явном виде энтропийные характеристики ряда ковариантных каналов. Более того, в случае аддитивности $C=C_{\chi}$ дает явное выражение для классической пропускной способности канала.

5.4.1. Унитальные кубитные каналы. Унитальные кубитные каналы (19) ковариантны относительно проективного представления группы $\mathbb{Z}_{2} \times \mathbb{Z}_{2}$, определяемого соотношениями

$$
U_{00}=\sigma_{0}, \quad U_{01}=\sigma_{z}, \quad U_{10}=\sigma_{x}, \quad U_{11}=-i \sigma_{y} .
$$

Поэтому соотношение (72) выполняется для этого класса каналов.

Вычислим энтропийные харктеристики унитального кубитного канала $\Phi$.

При вычислении $\check{H}(\Phi)$, принимая во внимание унитарную инвариантность квантовой энтропии, достаточно рассмотреть случай $\Phi=\Lambda$, где $\Lambda$ дается соотношением (17). Энтропия кубитного состояния (14), которое имеет собственные значения $\frac{1 \pm|\mathbf{a}|}{2}$, равна

$$
H(S)=h_{2}\left(\frac{1-|\mathbf{a}|}{2}\right) .
$$

Единичный шар кубитных состояний сжимается каналом $\Lambda$ в эллипсоид с длинами полуосей $\left|\lambda_{\gamma}\right|, \gamma=x, y, z$, и минимальная выходная энтропия достигается на самой длинной полуоси, что соответствует состоянию с собственными значениями $\frac{1 \pm \max _{\gamma}\left|\lambda_{\gamma}\right|}{2}$. Это дает

$$
\begin{gathered}
\nu_{p}(\Phi)=\left[\left(\frac{1-\max _{\gamma}\left|\lambda_{\gamma}\right|}{2}\right)^{p}+\left(\frac{1+\max _{\gamma}\left|\lambda_{\gamma}\right|}{2}\right)^{p}\right]^{1 / p}, \\
\check{H}(\Phi)=h_{2}\left(\frac{1-\max _{\gamma}\left|\lambda_{\gamma}\right|}{2}\right) .
\end{gathered}
$$

Применяя лемму 4, получаем

$$
C_{\chi}(\Phi)=\log 2-h_{2}\left(\frac{1-\max _{\gamma}\left|\lambda_{\gamma}\right|}{2}\right) .
$$


Используя разложение в выпуклую комбинацию диагональных каналов специального вида и применяя к ним неравенство Либа-Тирринга, К. Кинг [43] доказал выполнимость гипотезы (21) для всех $p>1$, а также гипотез (25) и (27) для случая, когда $\Phi_{1}$ - произвольный унитальный кубитный канал, а $\Phi_{2}$ - произвольный канал. Позитивные результаты для не-унитальных кубитных каналов получены в недавней работе [44].

5.4.2. Деполяризующий канал. Следующий пример - деполяризующий канал в $\mathscr{H}$

$$
\Phi(\rho)=(1-p) \rho+p \frac{I}{d} \operatorname{Tr} \rho, \quad 0 \leqslant p \leqslant \frac{d^{2}}{d^{2}-1} .
$$

Если $p \leqslant 1$, то это соотношение описывает смесь идеального канала Id и полностью деполяризующего канала. Для всего диапазона значений $0 \leqslant p \leqslant \frac{d^{2}}{d^{2}-1}$ полная положительность может быть доказана, используя представление Крауса (см., например, [1]). Деполяризующий канал характеризуется свойством унитарной ковариантности

$$
\Phi\left(U \rho U^{*}\right)=U \Phi(\rho) U^{*}
$$

для произвольного унитарного оператора $U$ в $\mathscr{H}$.

Для любого чистого состояния $\rho$ оператор $\Phi(\rho)$ имеет собственное значение $p / d$ кратности $(d-1)$ и простое собственное значение $\left(1-p \frac{d-1}{d}\right)$, откуда

$$
\nu_{p}(\Phi)=\left[\left(1-p \frac{d-1}{d}\right)^{p}+(d-1)\left(\frac{p}{d}\right)^{p}\right]^{1 / p}
$$

и

$$
\check{H}(\Phi)=-\left(1-p \frac{d-1}{d}\right) \log \left(1-p \frac{d-1}{d}\right)-p \frac{d-1}{d} \log \frac{p}{d} .
$$

$\chi$-пропускная способность дается формулой (72) и достигается на ансамбле равновероятных чистых состояний, соответствующих векторам ортонормированного базиса в $\mathscr{H}$.

Свойства (21) для всех $p>1,(25)$ и (27) были доказаны для случая, когда $\Phi_{1}$ - деполяризующий канал, а $\Phi_{2}$ - произвольный канал, в работе К. Кинга [45] с использованием метода, аналогичного случаю унитальных кубитных каналов.

Комплементарный к деполяризующему каналу вычислен в работе [46].

5.4.3. Транспонированно-деполяризующий канал. Рассмотрим подробно транспонированно-деполяризующий канал

$$
\Phi(\rho)=\frac{1}{d-1}\left[I \operatorname{Tr} \rho-\rho^{T}\right]
$$

где $\rho^{T}$ обозначает транспонирование $\rho$ в фиксированном ортонормированном базисе $\left\{e_{j}\right\}$ в $\mathscr{H}=\mathscr{H}_{A}=\mathscr{H}_{B}, \operatorname{dim} \mathscr{H}=d$. Полная положительность отображения (79) вытекает из представления Крауса

$$
\Phi(\rho)=\frac{1}{2(d-1)} \sum_{j, k=1}^{d}\left(\left|e_{j}\right\rangle\left\langle e_{k}|-| e_{k}\right\rangle\left\langle e_{j}\right|\right) \rho\left(\left|e_{k}\right\rangle\left\langle e_{j}|-| e_{j}\right\rangle\left\langle e_{k}\right|\right) .
$$


Этот канал интересен в том отношении, что он нарушает свойство мультипликативности (21) с $\Phi_{1}=\Phi_{2}=\Phi$ при $d \geqslant 3$ и достаточно больших $p$ [47]. $\mathrm{B}$ то же время мультипликативность имеет место при $1 \leqslant p \leqslant 2$ [48], как и аддитивность (25), см. [49], [50]. Он обладает свойством ковариантности

$$
\Phi\left(U \rho U^{*}\right)=\bar{U} \Phi(\rho) \bar{U}^{*}
$$

для произвольного унитарного $U$, где $\bar{U}$ обозначает комплексное сопряжение в базисе $\left\{e_{j}\right\}$. Отсюда следует, что для этого канала имеет место соотношение (72).

Для любого чистого входного состояния $\rho$ выход имеет вид $\Phi(\rho)=P /(d-1)$, где $P-(d-1)$-мерный проектор. Поэтому

$$
\nu_{p}(\Phi)=(d-1)^{1 / p-1} ; \quad \check{R}_{p}(\Phi)=\check{H}(\Phi)=\log (d-1) .
$$

Согласно лемме 4,

$$
C_{\chi}=\log d-\check{H}(\Phi)=\log \frac{d}{d-1} .
$$

С другой стороны, рассмотрим действие канала $\Phi \otimes \Phi$ на чистое состояние $\left|\psi_{12}\right\rangle\left\langle\psi_{12}\right|$. В силу свойства унитарной ковариантности, мы можем ограничиться случаем

$$
\left|\psi_{12}\right\rangle=\sum_{j=1}^{d} \sqrt{\lambda_{j}}\left|e_{j}\right\rangle \otimes\left|e_{j}\right\rangle
$$

где $\lambda_{j} \geqslant 0, \sum_{j=1}^{d} \lambda_{j}=1$. Тогда

$$
(\Phi \otimes \Phi)\left(\left|\psi_{12}\right\rangle\left\langle\psi_{12}\right|\right)=\frac{1}{(d-1)^{2}}\left[I_{12}-I_{1} \otimes \rho_{2}-\rho_{1} \otimes I_{2}+\left|\psi_{12}\right\rangle\left\langle\psi_{12}\right|\right],
$$

где $\rho_{1}=\rho_{2}=\sum_{j=1}^{d} \lambda_{j}\left|e_{j}\right\rangle\left\langle e_{j}\right|$. В частности, для максимально сцепленного входного состояния, $\lambda_{j}=1 / \sqrt{d}$, мы получаем выходное состояние

$$
(\Phi \otimes \Phi)\left(\left|\Omega_{12}\right\rangle\left\langle\Omega_{12}\right|\right)=\frac{1}{(d-1)^{2}}\left[\left(1-\frac{2}{d}\right) I_{12}+\left|\Omega_{12}\right\rangle\left\langle\Omega_{12}\right|\right],
$$

которое имеет собственное значение $\frac{1}{(d-1)^{2}}\left(1-\frac{2}{d}\right)$ кратности $d^{2}-1$ и простое собственное значение $\frac{1}{(d-1)^{2}}\left(2-\frac{2}{d}\right)$. Поэтому

$$
\begin{aligned}
\nu_{p}(\Phi \otimes \Phi)^{p} & \geqslant\left(d^{2}-1\right)\left(\frac{1-2 / d}{(d-1)^{2}}\right)^{p}+\left(\frac{2-2 / d}{(d-1)^{2}}\right)^{p} \\
& =\nu_{p}(\Phi)^{2 p}\left[\frac{d^{2}-1}{(d-1)^{2}}\left(1-\frac{2}{d}\right)^{p}+\frac{1}{(d-1)^{2}}\left(2-\frac{2}{d}\right)^{p}\right] .
\end{aligned}
$$

Если $d \geqslant 3$, то второе слагаемое в квадратных скобках стремится к бесконечности при $p \rightarrow \infty$. В частности, для $d=3$ оно превосходит 1 при $p>4.7823$ [47], таким образом нарушая мультипликативность

$$
\nu_{p}(\Phi \otimes \Phi)=\nu_{p}(\Phi)^{2} .
$$


С другой стороны, это свойство выполняется для всех $1 \leqslant p \leqslant 2$. Докажем это сначала для $p=2$. Из (81) получаем

$$
\operatorname{Tr}\left[(\Phi \otimes \Phi)\left(\left|\psi_{12}\right\rangle\left\langle\psi_{12}\right|\right)\right]^{2}=\frac{1}{(d-1)^{4}}\left[d^{2}-4 d+5+(2 d-4) \operatorname{Tr} \rho_{1}^{2}\right],
$$

что принимает максимальное значение, когда $\operatorname{Tr} \rho_{1}^{2}=1$, причем это максимальное значение равно $\frac{1}{(d-1)^{2}}=\nu_{2}(\Phi)^{4}$. Для $p \leqslant 2$ имеем, используя монотонность энтропии Реньи относительно параметра $p$ :

$$
2 \check{R}_{p}(\Phi)=2 \check{R}_{2}(\Phi)=\check{R}_{2}(\Phi \otimes \Phi) \leqslant \check{R}_{p}(\Phi \otimes \Phi)
$$

и противоположное неравенство очевидно. Переходя к пределу $p \downarrow 1$, получаем соответствующее свойство аддитивности минимальной выходной энтропии $\check{H}(\Phi)$. Мы намеренно ограничиваемся здесь этим элементарным рассуждением, которое, однако, может быть существенно обобщено как на более широкие классы каналов, так и на более общие формы свойства мультипликативности/аддитивности, см. [49], [50], [51], [52]. В частности, доказано, что $C=C_{\chi}=\log \frac{d}{d-1}$.

Этот пример также показывает, что хотя неравенство Либа-Тирринга может быть использовано в ряде случаев для доказательства свойства мультипликативности при всех $p \geqslant 1$, оно вряд ли может быть основой общего доказательства свойств аддитивности либо мультипликативности только при $1 \leqslant p \leqslant 2$. Более того, до сих пор нет единого доказательства, которое покрывало бы все рассмотренные выше случаи, так как в каждом из этих случаев применение неравенства Либа-Тирринга сопровождалось аргументами, специфическими для каждого случая.

Используя формулу (49) и представление Крауса (80), можно найти комплементарный канал

$$
\widetilde{\Phi}(\rho)=\frac{2}{(d-1)} P_{-}\left(\rho \otimes I_{2}\right) P_{-},
$$

который обладает теми же свойствами мультипликативности/аддитивности, что и канал (79) (см. подробнее [30]). Здесь $P_{-}$- проектор на антисимметричное подпространство пространства $\mathscr{H} \otimes \mathscr{H}$, которое имеет размерность $\frac{d(d-1)}{2}$. Свойство ковариантности канала (84) имеет вид

$$
\widetilde{\Phi}\left(U \rho U^{*}\right)=(U \otimes U) \widetilde{\Phi}(\rho)\left(U^{*} \otimes U^{*}\right),
$$

как следует из соотношения $P_{-}(U \otimes U)=(U \otimes U) P_{-}$. Согласно лемме 4 , пропускная способность этого канала равна

$$
C=C_{\chi}=\log \frac{d(d-1)}{2}-\check{H}(\Phi)=\log \frac{d}{2} .
$$

\section{6. Глобальная эквивалентность различных форм аддитивности}

В работе П. Шора [28] было дано доказательство ряда утверждений, связывающих различные свойства аддитивности; некоторые импликации на самом 
деле избыточны, хотя их доказательство представляет самостоятельный интерес. Ниже мы даем сокращенную и модифицированную версию, которая в сочетании с предложением 2 достаточна для доказательства эквивалентности различных свойств аддитивности.

ТЕОрема 4. Если аддитивность минималъной выходной энтропии (25) въполняется для всех каналов, то свойство (34) также выполняется для всех каналов.

ДокАЗАтельство. Прежде всего заметим, что $H_{\Phi}^{*}(X+\lambda I)=H_{\Phi}^{*}(X)+\lambda$, откуда следует, что достаточно установить (34) только для $X_{1}, X_{2} \leqslant 0$. Идея доказательства состоит в том, чтобы по данному каналу $\Phi$ и оператору $X \geqslant 0$ построить новый канал $\Phi_{X}^{\prime}$ (расширение Шора) такой, что

$$
\check{H}\left(\Phi_{X}^{\prime}\right)=\min _{\rho} H\left(\Phi_{X}^{\prime}(\rho)\right) \approx \min _{\rho}[H(\Phi(\rho))+\operatorname{Tr} \rho X]=-H_{\Phi}^{*}(-X) .
$$

Тогда мы можем применить аппарат двойственности из теории выпуклости для получения свойства аддитивности (34) для исходных каналов, что равносильно (30), из аддитивности минимальной выходной энтропии для расширений Шора исходных каналов.

Для данного канала $\Phi: \mathfrak{M}(\mathscr{H}) \rightarrow \mathfrak{M}\left(\mathscr{H}^{\prime}\right)$ и положительного $X \in \mathscr{H}$ новый канал $\Phi_{X}^{\prime}$ строится следующим образом: выберем постоянную $c \geqslant\|X\|$, тогда оператор $E=c^{-1} X$ удовлетворяет условию $0 \leqslant E \leqslant I$. Пусть числа $q_{n} \in(0,1)$ таковы, что

$$
\left(1-q_{n}\right) \log n=c, \quad n=2,3, \ldots
$$

Тогда канал $\Phi_{X}^{\prime}: \mathfrak{M}(\mathscr{H}) \rightarrow \mathfrak{M}\left(\mathscr{H}_{n}^{\prime}\right)$, где $\mathscr{H}_{n}^{\prime}=\mathscr{H}^{\prime} \oplus \mathscr{H}_{n} \oplus \mathbb{C}$, действует на состояние $\rho \in \mathfrak{M}(\mathscr{H})$ следующим образом:

$$
\Phi_{X}^{\prime}(\rho)=\left[\begin{array}{ccc}
q_{n} \Phi(\rho) & 0 & 0 \\
0 & \left(1-q_{n}\right)(\operatorname{Tr} \rho E) \frac{I_{n}}{n} & 0 \\
0 & 0 & \left(1-q_{n}\right) \operatorname{Tr} \rho(I-E)
\end{array}\right] .
$$

Это есть ортогональная выпуклая сумма в.п. отображений, сохраняющая след, т.е. канал. Мы называем $\Phi_{X}^{\prime}$ расширением Шора канала $\Phi$.

ПримечАниЕ. В основе этого определения лежат остроумные интуитивные соображения. Действие канала $\Phi_{X}^{\prime}(\rho)$ может быть описано следующим образом: с вероятностью $q_{n}$ (которая стремится к 1 при $n \rightarrow \infty$ ) он действует как исходный канал $\Phi$, давая состояние $\Phi(\rho)$. Однако с вероятностью $\left(1-q_{n}\right)$ вместо этого производится квантовое измерение, описываемое разложением единицы (квантовой наблюдаемой с двумя значениями) $\{E, I-E\}$, причем одно значение, например 1 , появляется с вероятностью $\operatorname{Tr} \rho E$, а другое, например $0,-$ с вероятностью $\operatorname{Tr} \rho(I-E)$. Если исход измерения - 1 , то на выходе появляется хаотическое состояние $I_{n} / n$ в $n$-мерном унитарном пространстве $\mathscr{H}_{n}$; если же исход - 0, то на выходе - чистое состояние, ортогональное пространству $\mathscr{H}^{\prime} \oplus \mathscr{H}_{n}$. Таким образом, канал $\Phi_{X}^{\prime}(\rho)$ с высокой вероятностью $q_{n}$ действует как исходный, однако с малой вероятностью $\left(1-q_{n}\right)(\operatorname{Tr} \rho E)$ выдает хаотическое состояние $I_{n} / n$ высокой размерности, что позволяет оценить значение $\operatorname{Tr} \rho E=c^{-1} \operatorname{Tr} \rho X$. 
Выходная энтропия канала $\Phi_{X}^{\prime}$ равна

$$
\begin{aligned}
H\left(\Phi_{X}^{\prime}(\rho)\right) & =q_{n} H(\Phi(\rho))+\left(1-q_{n}\right)(\operatorname{Tr} \rho E) \log n+h_{2}\left(q_{n}\right)+\left(1-q_{n}\right) h_{2}(\operatorname{Tr} \rho E) \\
& =q_{n} H(\Phi(\rho))+\operatorname{Tr} \rho X+o(1),
\end{aligned}
$$

где $o(1)$ стремится к 0 при $n \rightarrow \infty$ равномерно по $\rho$. Более того,

$$
H\left(\Phi_{X}^{\prime}(\rho)\right) \geqslant q_{n} H(\Phi(\rho))+\operatorname{Tr} \rho X .
$$

Теперь обозначим $\mathscr{H}=\mathscr{H}_{1}$, и пусть $\Psi: \mathfrak{M}\left(\mathscr{H}_{2}\right) \rightarrow \mathfrak{M}\left(\mathscr{H}_{2}^{\prime}\right)-$ другой произвольный канал, тогда $\Phi_{X}^{\prime} \otimes \Psi$ действует на состояние $\rho_{12} \in \mathfrak{M}\left(\mathscr{H}_{1} \otimes \mathscr{H}_{2}\right)$ согласно формуле

$$
\begin{aligned}
\left(\Phi_{X}^{\prime}\right. & \otimes \Psi)\left(\rho_{12}\right) \\
& =\left[\begin{array}{ccc}
q_{n}(\Phi \otimes \Psi)\left(\rho_{12}\right) & 0 & 0 \\
0 & \left(1-q_{n}\right)\left(\operatorname{Tr} \rho_{1} E\right)\left(\frac{I_{n}}{n} \otimes \sigma_{2}\right) & 0 \\
0 & 0 & \left(1-q_{n}\right)(\operatorname{Tr} \rho(I-E)) \sigma_{2}^{\prime}
\end{array}\right],
\end{aligned}
$$

где состояние $\sigma_{2} \in \mathfrak{S}\left(\mathscr{H}_{2}^{\prime}\right)$ определяется соотношением

$$
\sigma_{2}=\left(\operatorname{Tr} \rho_{1} E\right)^{-1} \operatorname{Tr}_{\mathscr{H}_{1}}\left(\sqrt{E} \otimes I_{2}\right)\left(\operatorname{Id}_{1} \otimes \Psi\right)\left(\rho_{12}\right)\left(\sqrt{E} \otimes I_{2}\right),
$$

а $\sigma_{2}^{\prime}$ - аналогичным соотношением с заменой $E$ на $I-E$ (если одна из вероятностей $\operatorname{Tr} \rho_{1} E, \operatorname{Tr} \rho(I-E)$ равна нулю, то соответствующее состояние выбирается произвольно). Из (88) получаем, аналогично (86),

$$
\begin{aligned}
H\left(\left(\Phi_{X}^{\prime} \otimes \Psi\right)\left(\rho_{12}\right)\right) & =q_{n} H\left((\Phi \otimes \Psi)\left(\rho_{12}\right)\right)+\left(1-q_{n}\right)(\operatorname{Tr} \rho E)\left(\log n+H\left(\sigma_{2}\right)\right)+o(1) \\
& =q_{n} H\left((\Phi \otimes \Psi)\left(\rho_{12}\right)\right)+\operatorname{Tr} \rho_{1} X+o(1)
\end{aligned}
$$

равномерно по $\rho_{12}$.

Пусть теперь $\Phi_{j} ; j=1,2,-$ какие-либо два канала, а $X_{j} \in \mathfrak{M}\left(\mathscr{H}_{j}\right)$ - два положительных оператора. Выбирая $c \geqslant \max \left\{\left\|X_{1}\right\|,\left\|X_{2}\right\|\right\}$, построим расширения Шора $\Phi_{1, X_{1}}^{\prime}, \Phi_{2, X_{2}}^{\prime}$. Применяя (89) дважды, находим

$$
H\left(\left(\Phi_{1, X_{1}}^{\prime} \otimes \Phi_{2, X_{2}}^{\prime}\right)\left(\rho_{12}\right)\right)=q_{n}^{2} H\left(\left(\Phi_{1} \otimes \Phi_{2}\right)\left(\rho_{12}\right)\right)+\operatorname{Tr} \rho_{1} X_{1}+\operatorname{Tr} \rho_{2} X_{2}+o(1),
$$

поэтому

$$
\begin{aligned}
\check{H}\left(\Phi_{1, X_{1}}^{\prime} \otimes \Phi_{2, X_{2}}^{\prime}\right) & =\min _{\rho_{12}}\left[q_{n}^{2} H\left(\left(\Phi_{1} \otimes \Phi_{2}\right)\left(\rho_{12}\right)\right)+\operatorname{Tr} \rho_{1} X_{1}+\operatorname{Tr} \rho_{2} X_{2}\right]+o(1) \\
& \leqslant \min _{\rho_{12}}\left[H\left(\left(\Phi_{1} \otimes \Phi_{2}\right)\left(\rho_{12}\right)\right)+\operatorname{Tr} \rho_{1} X_{1}+\operatorname{Tr} \rho_{2} X_{2}\right]+o(1) .
\end{aligned}
$$

Далее, из (87)

$$
\begin{aligned}
\check{H}\left(\Phi_{j, X_{j}}^{\prime}\right) & \geqslant \min _{\rho_{j}}\left[q_{n} H\left(\Phi_{j}\left(\rho_{j}\right)\right)+\operatorname{Tr} \rho_{j} X_{j}\right] \\
& \geqslant q_{n} \min _{\rho_{j}}\left[H\left(\Phi_{j}\left(\rho_{j}\right)\right)+\operatorname{Tr} \rho_{j} X_{j}\right] ; \quad j=1,2 .
\end{aligned}
$$


В силу предположения об аддитивности минимальной выходной энтропии,

$$
\check{H}\left(\Phi_{1, X_{1}}^{\prime} \otimes \Phi_{2, X_{2}}^{\prime}\right)=\check{H}\left(\Phi_{1, X_{1}}^{\prime}\right)+\check{H}\left(\Phi_{2, X_{2}}^{\prime}\right),
$$

поэтому

$$
-H_{\Phi_{1} \otimes \Phi_{2}}^{*}\left(-X_{1} \otimes I_{2}-I_{1} \otimes X_{2}\right)+o(1) \geqslant q_{n}\left[-H_{\Phi_{1}}^{*}\left(-X_{1}\right)-H_{\Phi_{2}}^{*}\left(-X_{2}\right)\right],
$$

и устремляя $n$ к $\infty$, получаем

$$
H_{\Phi_{1} \otimes \Phi_{2}}^{*}\left(-X_{1} \otimes I_{2}-I_{1} \otimes X_{2}\right) \leqslant H_{\Phi_{1}}^{*}\left(-X_{1}\right)+H_{\Phi_{2}}^{*}\left(-X_{2}\right),
$$

а следовательно, и (34). Теорема 4 доказана.

ПрЕДЛОЖЕНИЕ 10. Аддитивность ұ-пропускной способности (27) для всех каналов влечет аддитивность минимальной выходной энтропии (25) для всех каналов.

ДоказАтельство. По заданному каналу $\Phi: \mathfrak{M}(\mathscr{H}) \rightarrow \mathfrak{M}\left(\mathscr{H}^{\prime}\right)$ мы построим новый канал $\Phi^{\prime}$ такой, что

$$
\check{H}\left(\Phi^{\prime}\right)=\check{H}(\Phi) ; \quad C_{\chi}\left(\Phi^{\prime}\right)=\log d^{\prime}-\check{H}(\Phi) .
$$

Для этого мы всегда можем предположить, что в $\mathscr{H}^{\prime}$ имеется семейство унитарных операторов $\left\{W_{\gamma} ; \gamma \in G\right\}$ такое, что

$$
\frac{1}{N} \sum_{\gamma \in G} W_{\gamma} \rho W_{\gamma}^{*}=(\operatorname{Tr} \rho) \frac{I}{d^{\prime}} ; \quad \rho \in \mathfrak{M}\left(\mathscr{H}^{\prime}\right),
$$

где $N=|G|$. Например, $\left\{W_{\gamma}\right\}$ может быть неприводимым проективным представлением некоторой конечной группы, см. (73). Пусть $\mathscr{H}_{N}-N$ мерное унитарное пространство с ортонормированным базисом $\left\{e_{\gamma}\right\}$. Тогда канал $\Phi^{\prime}: \mathfrak{M}\left(\mathscr{H} \otimes \mathscr{H}_{N}\right) \rightarrow \mathfrak{M}\left(\mathscr{H}^{\prime}\right)$ действует на состояние $\sigma \in \mathfrak{S}\left(\mathscr{H} \otimes \mathscr{H}_{N}\right)$ следующим образом:

$$
\Phi^{\prime}(\sigma)=\frac{1}{N} \sum_{\gamma \in G} W_{\gamma} \Phi\left(\left\langle e_{\gamma}|\sigma| e_{\gamma}\right\rangle\right) W_{\gamma}^{*} .
$$

Следовательно, $\check{H}\left(\Phi^{\prime}\right) \geqslant \check{H}(\Phi)$, в силу вогнутости и унитарной инвариантности энтропии. С другой стороны, эта граница достигается, если положить $\sigma=$ $\rho_{0} \otimes\left|e_{\gamma_{0}}\right\rangle\left\langle e_{\gamma_{0}}\right|$, где $\rho_{0}-$ состояние, минимизирующее $H(\Phi(\rho))$, и $\gamma_{0} \in G$. Таким образом, мы получаем первое равенство в (90). В силу неравенства (28),

$$
C_{\chi}\left(\Phi^{\prime}\right) \leqslant \log d^{\prime}-\check{H}\left(\Phi^{\prime}\right)=\log d^{\prime}-\check{H}(\Phi),
$$

и эта граница достигается на ансамбле состояний $\sigma_{\gamma}=\rho_{0} \otimes\left|e_{\gamma}\right\rangle\left\langle e_{\gamma}\right|$, взятых с равными вероятностями $1 / N$, как видно из (91). Это доказывает второе равенство в (90).

Более того, аналогичное рассуждение применимо и к тензорному произведению каналов $\Phi_{1} \otimes \Phi_{2}$, снабженному семейством унитарных операторов $\left\{W_{\gamma}^{(1)} \otimes W_{\gamma}^{(2)}\right\}$ в $\mathscr{H}_{1}^{\prime} \otimes \mathscr{H}_{2}^{\prime}$, так что $\left(\Phi_{1} \otimes \Phi_{2}\right)^{\prime}=\Phi_{1}^{\prime} \otimes \Phi_{2}^{\prime}$ и

$$
\begin{gathered}
\check{H}\left(\Phi_{1}^{\prime} \otimes \Phi_{2}^{\prime}\right)=\check{H}\left(\Phi_{1} \otimes \Phi_{2}\right) ; \\
C_{\chi}\left(\Phi_{1}^{\prime} \otimes \Phi_{2}^{\prime}\right)=\log d_{1}^{\prime} d_{2}^{\prime}-\check{H}\left(\Phi_{1} \otimes \Phi_{2}\right) .
\end{gathered}
$$


Следовательно, аддитивность $\chi$-пропускной способности $(27)$ для $\Phi_{1}^{\prime}, \Phi_{2}^{\prime}$ влечет аддитивность минимальной выходной энтропии $(25)$ для $\Phi_{1}, \Phi_{2}$. Предложение 10 доказано.

\section{7. Бесконечномерные каналы}

Мы видели выше, что проблема аддитивности остается открытой даже для минимальной размерности 2: неизвестно, выполняются ли свойства аддитивности для всех не-унитальных кубитных каналов, хотя в пользу этого имеются убедительные численные и аналитические данные (см. [53], [54], [44]). Тем не менее, есть ряд важных оснований для рассмотрения этой проблемы и в бесконечномерных пространствах.

Имеется важный и интересный класс бозонных гауссовских каналов (см. [55]), которые действуют в бесконечномерном гильбертовом пространстве. Исследование свойств непрерывности энтропийных характеристик бесконечномерных каналов становится нетривиальным, поскольку, как известно, энтропия приобретает некоторые патологические свойства. В бесконечномерном случае она лишь полунепрерывна снизу и "почти всюду" бесконечна [56]. Другим важным вопросом становится получение условий компактности подмножеств квантовых состояний и ансамблей, существенных для достижимости экстремумов в выражениях для пропускной способности и выпуклого замыкания выходной энтропии.

Доказательство глобальной эквивалентности различных форм аддитивности для конечномерных каналов (раздел 6), использующее расширения неограниченно возрастающей размерности, на самом деле, опирается на разрывность $\chi$ пропускной способности как функции канала в бесконечной размерности. Это также требует внимательного изучения свойств непрерывности энтропийных характеристик бесконечномерных каналов. Такое исследование было предпринято в серии работ [57]-[59]. В частности, было показано, что несмотря на упомянутое выше отсутствие непрерывности, выполнение гипотезы аддитивности для всех конечномерных каналов влечет аддитивность $\chi$-пропускной способности бесконечномерных каналов с произвольными ограничениями на входе [59].

Для каналов бесконечной размерности характерны две важные особенности. Одна состоит в необходимости ограничений на входе (таких как ограничение на мощность сигнала для гауссовских каналов), чтобы избежать бесконечных значений пропускной способности (хотя рассмотрение ограничений на входе оказалось полезным и при изучении гипотезы аддитивности для конечномерных каналов [40]). Другая особенность состоит в том, что естественно возникают бесконечные и, как правило, "непрерывные" ансамбли состояний, понимаемые как вероятностные меры на множестве всех квантовых состояний. Используя критерии компактности, известные в теории вероятностей и в теории операторов, можно показать, что множество всех таких обобщенных ансамблей с барицентрами в компактном множестве состояний само является слабо компактным. Опираясь на этот факт, можно получить достаточное условие существования оптимального обобщенного ансамбля. Это условие эффективно проверяется для бозонных гауссовских каналов с ограничением на мощность сигнала [58]. Однако, кроме самого факта существования, желательно было бы иметь явное описание оптимальных состояний и ансамблей в случае квантовых гауссовских каналов. В классической теории информации пропускная 
способность гауссовских каналов достигается на гауссовских распределениях, более того, имеются соответствующие аналитические результаты для норм интегральных операторов с гауссовскими ядрами (см. [6]). Имеет ли место аналог этого свойства для бозонных гауссовских каналов - еще одна открытая проблема, которая заслуживает отдельного обсуждения (относительно некоторых частичных результатов в этом направлении см. [55], [60]-[62]). Мы только отметим, что положительное решение этой проблемы, по-видимому, также зависит от выполнимости гипотезы мультипликативности/аддитивности [6], [62].

\section{8. Приложение: о $q \rightarrow p$ нормах в.п. отображений}

ПрЕДЛОЖЕНИЕ 11. Величина максимума в определении (12) нормы $\|\Phi\|_{q \rightarrow p}$ в.п. отображения $\Phi$ остается неизменной, если вместо всех $F \neq 0$ максимум берется толъко по всем эрмитовым $F \geqslant 0$. В частности, для $q=1$ имеет место соотношение (20).

ДокАЗАТЕЛьСтво. Возможность сведения к эрмитовым $F$ была впервые установлена в [22], но мы будем следовать более простым аргументам работы [23]. Мы хотим доказать, что

$$
\|\Phi\|_{q \rightarrow p}^{p}=\max _{\substack{F \geqslant 0 \\ \operatorname{Tr}|\Phi(F)|^{q}=1}} \operatorname{Tr}|\Phi(F)|^{p} \equiv \nu_{q \rightarrow p}(\Phi)^{p} .
$$

Пусть $F=U|F|$ - полярное разложение оператора $F$, тогда $\sqrt{F F^{*}}=U|F| U^{*}$. Рассмотрим блочный эрмитов оператор в $\mathscr{H} \oplus \mathscr{H}$ вида

$$
G=\left[\begin{array}{cc}
0 & F \\
F^{*} & 0
\end{array}\right], \quad \text { так что } \quad|G|=\left[\begin{array}{cc}
U|F| U^{*} & 0 \\
0 & |F|
\end{array}\right]
$$

Используя изоморфизм $\mathscr{H} \oplus \mathscr{H} \simeq \mathscr{H} \otimes \mathbb{C}^{2}$, имеем

$$
(\Phi \otimes \mathrm{Id})(G)=\left[\begin{array}{cc}
0 & \Phi(F) \\
\Phi(F)^{*} & 0
\end{array}\right], \quad(\Phi \otimes \mathrm{Id})(|G|)=\left[\begin{array}{cc}
\Phi\left(U|F| U^{*}\right) & 0 \\
0 & \Phi(|F|)
\end{array}\right]
$$

где Id - тождественный канал. Здесь мы также использовали эрмитовость положительного отображения $\Phi$.

Поскольку $\Phi$ - в.п. отображение, отображение $\Psi=\Phi \otimes \operatorname{Id}$ положительно. В работе [12] было показано, что для положительного отображения $\Psi$ и эрмитова оператора $G$

$$
\operatorname{Tr}|\Psi(G)|^{p} \leqslant \operatorname{Tr} \Psi(|G|)^{p} ; \quad p \geqslant 1
$$

В самом деле, пусть $G=G_{+}-G_{-}$- разложение эрмитова оператора $G$ на положительную и отрицательную части, тогда $|G|=G_{+}+G_{-}$и $-\Psi(|G|) \leqslant \Psi(G) \leqslant$ $\Psi(|G|)$ в силу положительности $\Psi$. Для доказательства (92) обозначим $\left\{e_{j}\right\}$ базис из собственных векторов оператора $\Psi(G)$, а $\left\{f_{k}\right\}$ - базис из собственных 
векторов оператора $\Psi(|G|)$, тогда имеем

$$
\begin{aligned}
\operatorname{Tr}|\Psi(G)|^{p} & =\sum_{j}\left|\left\langle e_{j}|\Psi(G)| e_{j}\right\rangle\right|^{p} \leqslant \sum_{j}\left\langle e_{j}|\Psi(|G|)| e_{j}\right\rangle^{p} \\
& =\sum_{j}\left(\sum_{k}\left|\left\langle e_{j} \mid f_{k}\right\rangle\right|^{2}\left\langle f_{k}|\Psi(|G|)| f_{k}\right\rangle\right)^{p} \\
& \leqslant \sum_{j} \sum_{k}\left|\left\langle e_{j} \mid f_{k}\right\rangle\right|^{2}\left\langle f_{k}|\Psi(|G|)| f_{k}\right\rangle^{p}=\operatorname{Tr} \Psi(|G|)^{p},
\end{aligned}
$$

откуда и следует неравенство (92).

Теперь находим

$$
\begin{aligned}
\operatorname{Tr}|(\Phi \otimes \operatorname{Id})(G)|^{p} & =\operatorname{Tr}\left|\left[\begin{array}{cc}
0 & \Phi(F) \\
\Phi(F)^{*} & 0
\end{array}\right]\right|^{p} \\
& =\operatorname{Tr}\left|\left[\begin{array}{cc}
\sqrt{\Phi(F) \Phi(F)^{*}} & 0 \\
0 & \sqrt{\Phi(F)^{*} \Phi(F)}
\end{array}\right]\right|^{p} \\
& =2 \operatorname{Tr}|\Phi(F)|^{p},
\end{aligned}
$$

где мы использовали унитарную эквивалентность операторов $A A^{*}$ и $A^{*} A$. Аналогично

$$
\begin{aligned}
\operatorname{Tr}(\Phi \otimes \operatorname{Id})(|G|)^{p} & =\operatorname{Tr}\left|\left[\begin{array}{cc}
0 & \Phi(F) \\
\Phi(F)^{*} & 0
\end{array}\right]\right|^{p} \\
& =\operatorname{Tr}\left|\left[\begin{array}{cc}
\Phi\left(U|F| U^{*}\right) & 0 \\
0 & \Phi(|F|)
\end{array}\right]\right|^{p} \\
& =\operatorname{Tr} \Phi\left(U|F| U^{*}\right)^{p}+\operatorname{Tr} \Phi(|F|)^{p} .
\end{aligned}
$$

Из (92) получаем

$$
2 \operatorname{Tr}|\Phi(F)|^{p} \leqslant \operatorname{Tr} \Phi\left(U|F| U^{*}\right)^{p}+\operatorname{Tr} \Phi(|F|)^{p} .
$$

Из определения следует, что правая часть не превосходит $2 \nu_{q \rightarrow p}(\Phi)^{p}\left[\operatorname{Tr}|F|^{q}\right]^{p / q}$, откуда следует, что $\|\Phi\|_{q \rightarrow p} \leqslant \nu_{q \rightarrow p}(\Phi)$, тогда как противоположное неравенство очевидно. Предложение 11 доказано.

Работа поддержана научной программой Отделения математики РАН. Эта статья была начата, когда автор был приглашенным профессором в Отделении прикладной математики и теоретической физики Кембриджского университета. Автор благодарен Ю. М. Сухову, Н. Датта и М. Е. Широкову за полезные обсуждения.

\section{Список литературы}

[1] А. С. Холево, Введение в квантовую теорию информации, МЦНМО, М., 2002.

[2] C. H. Bennett, P. W. Shor, "Quantum information theory", IEEE Trans. Inform. Theory, 44:6 (1998), 2724-2742.

[3] M. A. Nielsen, I. L. Chuang, Quantum computation and quantum information, Cambridge Univ. Press, Cambridge, 2000. 
[4] W. Beckner, "Inequalities in Fourier analysis", Ann. of Math. (2), 102:1 (1975), 159182.

[5] G. Bennett, "Schur multipliers", Duke Math. J., 44:3 (1977), 603-639.

[6] E. H. Lieb, "Gaussian kernels have only Gaussian maximizers", Invent. Math., 102:1 (1990), 179-208.

[7] M. Lifshits, A. Nazarov, Ya. Nikitin, "Tail behavior of anisotropic norms for Gaussian random fields", C. R. Math. Acad. Sci. Paris, 336:1 (2003), 85-88.

[8] R. Schatten, Norm ideals of completely continuous operators, Springer-Verlag, Berlin, 1960.

[9] M.-D. Choi, "Completely positive maps on complex matrices", Linear Algebra Appl., 10 (1975), 285-290.

[10] А. И. Кострикин, Ю. И. Манин, Линейная алгебра и геометрия, Наука, М., 1986.

[11] Г. Г. Амосов, А. С. Холево, Р. Ф. Вернер, "О некоторых проблемах аддитивности в квантовой теории информации", Проблемъ передачи информаиии, 36:4 (2000), 25-34; arXiv: math-ph/0003002.

[12] Г. Г. Амосов, А.С. Холево, "О гипотезе мультипликативности для квантовых каналов", Теория вероян. и ее примен., 47:1 (2002), 143-146; arXiv:math-ph/ 0103015

[13] E. A. Carlen, E. H. Lieb, "A Minkowsky type trace inequality and strong subadditivity of quantum entropy", Differential operators and spectral theory, Amer. Math. Soc. Transl. (2), 189, Amer. Math. Soc., Providence, RI, 1999, 59-68.

[14] G. Pisier, Non-commutative vector valued $L_{p}$-spaces and completely $p$-summing maps, Astérisque, 247, Société Mathématique de France, Paris, 1998.

[15] I. Devetak, M. Junge, C. King, M. B. Ruskai, "Multiplicativity of completely bounded p-norms implies a new additivity result", arXiv: quant-ph/0506196.

[16] W. F. Stinespring, "Positive functions on $C^{*}$-algebras", Proc. Amer. Math. Soc., 6 (1955), 211-216.

[17] C. King, M. B. Ruskai, "Comments on multiplicativity of maximal p-norms when $p=2$ ", Quantum information, statistics, probability, ed. O. Hirota, Rinton Press, Princeton, NJ, 2004, 102-114; arXiv: quant-ph/0401026.

[18] C. King, M. Nathanson, M. B. Ruskai, "Multiplicativity properties of entrywise positive maps on matrix algebras", Linear Algebra Appl., 404 (2005), 367-379; arXiv: quant-ph/0409181.

[19] Е. А. Морозова, Н. Н. Ченцов, "Марковская инвариантная геометрия на многообразиях состояний", Н.Н. Ченцов. Избранные труды. Математика, Физматлит, M., 2001, 234-265.

[20] M. B. Ruskai, S. Szarek, E. Werner, "An analysis of completely positive trace-preserving maps on $\mathscr{M}_{2}$ matrices", Linear Algebra Appl., 347 (2002), 159-187; arXiv: quant-ph/0101003.

[21] M. Fukuda, A. S. Holevo, "On Weyl-covariant channels", arXiv: quant-ph/0510148.

[22] J. Watrous, "Notes on super-operator norms induced by Schatten norms", Quantum Inf. Comput., 5:1 (2005), 58-68; arXiv: quant-ph/0411077.

[23] K. M.R. Audenaert, "A note on the $p \rightarrow q$ norms of completely positive maps", arXiv: math-ph/0505085.

[24] B. Schumacher, M. D. Westmoreland, "Optimal signal ensembles", Phys. Rev. A, 63 (2001), 022308; arXiv: quant-ph/9912122.

[25] C. H. Bennett, C. A. Fuchs, J. A. Smolin, "Entanglement-enhanced classical communication on a noisy quantum channel", Quantum communication, computing and measurement, Proc. QCM96, ed. O. Hirota, A. S. Holevo, and C. M. Caves, Plenum, New York, 1997, 79-88; arXiv: quant-ph/9611006.

[26] А. С. Холево, “Квантовые теоремы кодирования", УМН, 53:6 (1998), 193-230. 
[27] Г. Г. Магарил-Ильяев, В. М. Тихомиров, Выпуклый анализ и его приложения, Эдиториал УРСС, М., 2000.

[28] P. W. Shor, "Equivalence of additivity questions in quantum information theory", Comm. Math. Phys., 246:3 (2004), 453-472; arXiv: quant-ph/0305035.

[29] K. M. R. Audenaert, S. L. Braunstein, "On strong superadditivity of the entanglement of formation", Comm. Math. Phys., 246:3 (2004), 443-452; arXiv: quant-ph/ 0303045.

[30] A.S. Holevo, "On complementary channels and the additivity problem", arXiv: quant-ph/0509101.

[31] C. King, K. Matsumoto, M. Natanson, M. B. Ruskai, "Properties of conjugate channels with applications to additivity and multiplicativity", arXiv: quant-ph/0509126.

[32] M. Fukuda, "Extending additivity from symmetric to asymmetric channels", arXiv: quant-ph/0505022.

[33] M. Horodecki, P. W. Shor, M. B. Ruskai, "Entanglement breaking channels", Rev. Math. Phys., 15:6 (2003), 629-641; arXiv: quant-ph/0302031.

[34] C. King, "An application of a matrix inequality in quantum information theory", arXiv: quant-ph/0412046.

[35] P. W. Shor, "Additivity of the classical capacity of entanglement-breaking quantum channels", J. Math. Phys., 43:9 (2002), 4334-4340; arXiv: quant-ph/0201149.

[36] C. King, "Maximal p-norms of entanglement breaking channels", Quantum Inf. Comput., 3:2 (2003), 186-190; arXiv: quant-ph/0212057.

[37] E. H. Lieb, W.E. Thirring, "Inequalities for the moments of the eigenvalues of the Schrödinger Hamiltonian and their relation to Sobolev inequalities", Studies in mathematical physics, ed. E. H. Lieb, B. Simon, and A. Wightman, Princeton Univ. Press, Princeton, 1976, 269-297.

[38] R. Bhatia, Matrix analysis, Graduate Texts Math., 169, Springer-Verlag, New York, 1997.

[39] H. Araki, "On an inequality of Lieb and Thirring", Lett. Math. Phys., 19:2 (1990), $167-170$.

[40] A. S. Holevo, M. E. Shirokov, "On Shor's channel extension and constrained channels", Comm. Math. Phys., 249:2 (2004), 417-430; arXiv: quant-ph/0306196.

[41] G. W. Mackey, Unitary group representations in physics, probability and number theory, Benjamin / Cummings, Reading, MA, 1978.

[42] A.S. Holevo, "A note on covariant dynamical semigroups", Rep. Math. Phys., 32:2 (1993), 211-216.

[43] C. King, "Additivity for unital qubit channels", J. Math. Phys., 43:10 (2002), 46414653; arXiv: quant-ph/0103156.

[44] C. King, N. Koldan, "New multiplicativity results for qubit maps", arXiv: quant-ph/ 0512185.

[45] C. King, "The capacity of the quantum depolarizing channel", IEEE Trans. Inform. Theory, 49:1 (2003), 221-229; arXiv: quant-ph/0204172.

[46] N. Datta, A. S. Holevo, "Complementarity and additivity for depolarizing channels", arXiv: quant-ph/0510145.

[47] R. F. Werner, A. S. Holevo, "Counterexample to an additivity conjecture for output purity of quantum channels", J. Math. Phys., 43:9 (2002), 4353-4357.

[48] N. Datta, "Multiplicativity of maximal $p$-norms in Werner-Holevo channels for $1 \leqslant$ $p \leqslant 2 "$, arXiv: quant-ph/0410063.

[49] K. Matsumoto, F. Yura, "Entanglement cost of antisymmetric states and additivity of capacity of some quantum channel", J. Phys. A, 37:15 (2004), L167-L171; arXiv: quant-ph/0306009. 
[50] N. Datta, A.S. Holevo, Y.M. Suhov, "A quantum channel with additive minimum output entropy", arXiv: quant-ph/0408176.

[51] R. Alicki, M. Fannes, "Note on multiple additivity of minimal Renyi entropy output of the Werner-Holevo channels", Open Syst. Inf. Dyn., 11:4 (2004), 339-342; arXiv: quant-ph/0407033.

[52] M. M. Wolf, J. Eisert, "Classical information capacity of a class of quantum channels", arXiv: quant-ph/0412133.

[53] S. Osawa, H. Nagaoka, "Numerical experiments on the capacity of quantum channel with entangled input states", arXiv: quant-ph/0007115.

[54] M. Hayashi, H. Imai, K. Matsumoto, M. B. Ruskai, T. Shimono, "Qubit channels which require four inputs to achieve capacity: implications for additivity conjectures", Quantum Inf. Comput., 5:1 (2005), 13-31; arXiv: quant-ph/0403176.

[55] A.S. Holevo, R.F. Werner, "Evaluating capacities of Bosonic Gaussian channels", Phys. Rev. A, 63 (2001), 032312; arXiv: quant-ph/9912067.

[56] A. Wehrl, "General properties of entropy", Rev. Modern Phys., 50:2 (1978), 221-260.

[57] А. С. Холево, "Классические пропускные способности квантового канала с ограничением на входе", Теория вероятн. и ее примен., 48:2 (2003), 359-374.

[58] А. С. Холево, М. Е. Широков, "Непрерывные ансамбли и пропускная способность квантовых каналов бесконечной размерности", Теория вероятн. и ее примен., 50:1 (2005), 98-114; arXiv: quant-ph/0408176.

[59] M. E. Shirokov, "The Holevo capacity of infinite dimensional channels and the additivity problem", arXiv: quant-ph/0408009.

[60] V. Giovannetti, S. Lloyd, L. Maccone, J. H. Shapiro, B. J. Yen, "Minimum Rényi and Wehrl entropies at the output of bosonic channels", Phys. Rev. A (3), 70:2 (2004), 022328; arXiv: quant-ph/0404037.

[61] V. Giovannetti, S. Lloyd, "Additivity properties of a Gaussian channel", Phys. Rev. A (3), 69:6 (2004), 062307; arXiv: quant-ph/0403075.

[62] M. M. Wolf, G. Giedke, J. I. Cirac, "Extremality of Gaussian quantum states", arXiv: quant-ph/0509154.

А. С. Холево (А. S. Holevo)

Математический институт им. В. А. Стеклова РАН

E-mail: holevo@mi.ras.ru
Поступила в редакцию 12.01 .2006 